\title{
Microscopic origin of highly enhanced current carrying capabilities of thin $\mathrm{NdFeAs}(\mathrm{O}, \mathrm{F})$ films $\dagger$
}

Cite this: Nanoscale Adv., 2019, 1, 3036

\author{
Sandra Kauffmann-Weiss, (D) *a Kazumasa lida, (D) bc Chiara Tarantini, (DD d \\ Torben Boll, ${ }^{\text {ef Reinhard Schneider, }}{ }^{9}$ Taito Ohmura, ${ }^{c}$ Takuya Matsumoto, \\ Takafumi Hatano, ${ }^{\text {bc }}$ Marco Langer, ${ }^{a}$ Sven Meyer, ${ }^{a}$ Jan Jaroszynski, ${ }^{d}$ \\ Dagmar Gerthsen, ${ }^{9}$ Hiroshi Ikuta, ${ }^{b c}$ Bernhard Holzapfel ${ }^{a}$ and Jens Hänisch (DD ${ }^{a}$
}

\begin{abstract}
Fe-based superconductors present a large variety of compounds whose physical properties strongly depend on the crystal structure and chemical composition. Among them, the so-called 1111 compounds show the highest critical temperature $T_{c}$ in the bulk form. Here we demonstrate the realization of excellent superconducting properties in $\mathrm{NdFeAs}\left(\mathrm{O}_{1-x} \mathrm{~F}_{x}\right)$. We systematically investigated the correlation between the microstructure at the nanoscale and superconductivity in an epitaxial $22 \mathrm{~nm}$ $\mathrm{NdFeAs}\left(\mathrm{O}_{1-x} \mathrm{~F}_{x}\right)$ thin film on a $\mathrm{MgO}$ single crystalline substrate $\left(T_{\mathrm{c}}=44.7 \mathrm{~K}\right)$. Atomic resolution analysis of the microstructure by transmission electron microscopy and atom probe tomography identified several defects and other inhomogeneities at the nanoscale that can act as extrinsic pinning centers. X-Ray diffraction and transmission electron microscopy displayed a broad variation of the a-axis lattice parameter either due to a partially strained layer at the interface to the substrate, high local strain at dislocation arrays, mosaicity, or due to composition variation within the film. The electrical transport properties are substantially affected by intrinsic pinning and a matching field corresponding to the film thickness and associated with the Bean-Livingston surface barrier of the surfaces. The thin film showed a self-field critical current density $J_{\mathrm{c}}(4.2 \mathrm{~K})$ of $\sim 7.6 \mathrm{MA} \mathrm{cm}^{-2}$ and a record pinning force density of $F_{\mathrm{p}} \approx$ $1 \mathrm{TN} \mathrm{m}^{-3}$ near $35 \mathrm{~T}$ for $H \| a b$ at $4.2 \mathrm{~K}$. These investigations highlight the role of the microstructure in fine-tuning and possibly functionalizing the superconductivity of Fe-based superconductors.
\end{abstract}

Received 7th March 2019 Accepted 3rd June 2019

DOI: $10.1039 / c 9 n a 00147 f$

rsc.li/nanoscale-advances

\section{Introduction}

Fe-based superconductors (FBS) have been studied extensively during the last 10 years since they represent a new class of unconventional superconductors exhibiting the highest critical temperature $\left(T_{\mathrm{c}}\right)$ apart from cuprates. Due to their high $T_{\mathrm{c}}$ and upper critical field $\mu_{0} H_{\mathrm{c} 2}$ (estimated to $\sim 100 \mathrm{~T}$ at $0 \mathrm{~K}$ by using the Werthamer-Helfand-Hohenberg (WHH) model $\left.^{1}\right){ }^{2}$ these

aInstitute for Technical Physics (ITEP), Karlsruhe Institute of Technology (KIT), 76344 Eggenstein-Leopoldshafen, Germany. E-mail: sandra.kauffmann-weiss@kit.edu

${ }^{b}$ Department of Materials Physics, Nagoya University, Furo-cho, Chikusa-ku, Nagoya 464-8603, Japan

'Department of Crystalline Materials Science, Nagoya University, Furo-cho, Chikusaku, Nagoya 464-8603, Japan

${ }^{d}$ Applied Superconductivity Center, National High Magnetic Field Laboratory, Florida State University, 2031 East Paul Dirac Drive, Tallahassee, Florida 32310, USA

${ }^{e}$ Karlsruhe Nano Micro Facility (KNMF), Karlsruhe Institute of Technology (KIT), 76344, Eggenstein-Leopoldshafen, Germany

Institute for Applied Materials (IAM-WK), Karlsruhe Institute of Technology (KIT), 76344, Karlsruhe, Germany

${ }^{8}$ Laboratory for Electron Microscopy (LEM), Karlsruhe Institute of Technology (KIT), Engesserstraße 7, 76131 Karlsruhe, Germany

$\dagger$ Electronic supplementary information (ESI) available. See DOI: $10.1039 / \mathrm{c} 9 \mathrm{na00147f}$ materials are attractive for basic science and engineering, e.g. for high-field magnetic applications. The evaluation of highfield transport properties like $H_{\mathrm{c} 2}$ and critical current density $J_{\mathrm{c}}$ is therefore of interest because of the observed weak field dependence of $J_{\mathrm{c}}$ at low magnetic fields and the variety of physical properties which strongly depend on the structure and chemical composition in the different material classes. Relevant material classes are the so-called "11", "122" and "1111" compounds to name the most important ones for potential applications, where the figures in the classification are the elemental ratio or stoichiometry in the chemical formula of the compound. ${ }^{3}$

1111 compounds, i.e. mainly $\operatorname{LnFeAs}(\mathrm{O}, \mathrm{F})(\mathrm{Ln}=$ lanthanoid), which crystallize in the ZrCuSiAs structure, show the highest $T_{\mathrm{c}}$ values up to around $58 \mathrm{~K}$, measured in both SmFeAs(O,F) single crystals and thin films. ${ }^{4,5}$ The 1111 compounds (for an early review see Ivanovskii ${ }^{6}$ ) consist of two distinct layers in the unit cell (similar to $\mathrm{Nd}_{2} \mathrm{CuO}_{4}$ among cuprates) - a metallic conducting $\mathrm{Fe}_{2} \mathrm{As}_{2}$ layer and a $\mathrm{Ln}_{2} \mathrm{O}_{2}$ layer. When oxygen is partially replaced by fluorine, and hence excess electrons transfer to the $\mathrm{Fe}_{2} \mathrm{As}_{2}$ layers, superconductivity develops in these compounds. The growth of single crystals as well as thin films of these compounds was initially 
difficult. Nevertheless, relatively large single crystals (with a maximum of $2 \mathrm{~mm}^{3}$ for La1111 (ref. 7) or Sm1111 (ref. 8)) and epitaxial thin films ${ }^{9}$ have been realized, which opens the opportunity to study transport properties. Indeed, transport $J_{\mathrm{c}}$ measurements using 1111 thin films ${ }^{10-13}$ and single crystals ${ }^{\mathbf{1 4}}$ have been reported in the past. However, detailed information on the relationship between the microstructure and the transport properties of $\operatorname{LnFeAs}(\mathrm{O}, \mathrm{F})$ thin films is missing in the literature.

One interesting member of the 1111 family is $\operatorname{NdFeAs}\left(\mathrm{O}_{1-x} \mathrm{~F}_{x}\right)$ which shows promising superconducting properties, such as a similarly high $T_{\mathrm{c}}$ of up to $56 \mathrm{~K}$ and self-field critical current densities $J_{\mathrm{c}, \mathrm{sf}}$ of up to $3.3 \mathrm{MA} \mathrm{cm} \mathrm{cm}^{-2}$ at low temperatures. ${ }^{12,15}$ Epitaxial $\mathrm{NdFeAs}\left(\mathrm{O}_{1-x} \mathrm{~F}_{x}\right)$ films were grown by a two-step process using molecular beam epitaxy (MBE): the deposition of the mother compound NdFeAsO, followed by Fdiffusion either from a NdOF over-layer or from a fluorinecontaining substrate $\left(\right.$ e.g. $\left.\mathrm{CaF}_{2}\right) \cdot{ }^{\mathbf{1 5 , 1 6}}$

The purpose of this paper is to investigate how the structure of a thin $\mathrm{NdFeAs}\left(\mathrm{O}_{1-x} \mathrm{~F}_{x}\right)$ film influences its pinning properties and $J_{\mathrm{c}}$ taking into account different possible effects like chemical composition, stoichiometric homogeneity, growth defects and strain effects. Structural properties were characterized by using X-ray diffraction (XRD) and atomic resolution analysis methods like transmission electron microscopy (TEM) and atom probe tomography (APT). These methods directly resolve locally the stoichiometry on the nanometer scale. The electrical transport properties were characterized in a wide temperature range and in high static fields up to $35 \mathrm{~T}$. The precise knowledge of structure, orientation, and strain state in combination with nanoscale microstructural and compositional characterization enables us to understand what determines $J_{\mathrm{c}}$ and pinning force variation in $\operatorname{NdFeAs}\left(\mathrm{O}_{1-x} \mathrm{~F}_{x}\right)$.

\section{Experimental}

\section{Synthesis}

An epitaxial, $22 \mathrm{~nm} \operatorname{NdFeAs}(\mathrm{O}, \mathrm{F})$ thin film of this study was grown on a $\mathrm{MgO}(001)[100]$ single crystal by molecular beam epitaxy (MBE) using solid sources of $\mathrm{Fe}_{2} \mathrm{O}_{3}, \mathrm{NdF}_{3}, \mathrm{Fe}$, As and $\mathrm{Ga}$ and $\mathrm{Fe}_{2} \mathrm{O}_{3}$ as a source for $\mathrm{O}_{2}$ according to ref. 16. Ga works as a fluorine getter according to the following reaction: $\mathrm{Ga}+\mathrm{NdF}_{3}$ $\rightarrow \mathrm{Nd}+\mathrm{GaF}_{3} \uparrow$. First the mother compound NdFeAsO was deposited with a nominal thickness of $20 \mathrm{~nm}$, followed by a $15 \mathrm{~nm}$ NdOF cap layer. Both layers were deposited at $800{ }^{\circ} \mathrm{C}$. During the NdOF cap layer deposition, $F$ diffuses into the $\mathrm{NdFeAsO}$ layer. The deposition rate for both $\mathrm{NdFeAsO}$ and NdOF is approximately $1 \mathrm{~nm} \min ^{-1}$.

\section{Microstructural characterization}

To determine the crystal structure, X-ray $\theta-2 \theta$ scans in BraggBrentano geometry ( $0 \mathrm{D}$ detector) and wide-range reciprocal space mapping (WRRSM) in a symmetric configuration with a $2 \mathrm{D}$ detector were performed using two Rigaku systems $(\mathrm{Cu}$ radiation).
A TEM lamella was prepared by means of a focused ion beam (FIB) using an FEI DualBeam Strata 400S instrument. Before FIB preparation of the TEM lamella, a $\mathrm{Pt} / \mathrm{C}$ protection layer was deposited on the top of the sample. Structural and microchemical properties were investigated by conventional transmission electron microscopy (TEM), electron diffraction, and scanning TEM (STEM) in combination with energy-dispersive Xray spectroscopy (EDXS).

Conventional TEM and combined STEM/EDXS analyses were carried out on a $200 \mathrm{kV}$ FEI Tecnai Osiris microscope, whereas for HRTEM imaging an aberration-corrected ( $C_{\mathrm{s}}$ image corrector) $300 \mathrm{kV}$ FEI Titan 80-300 microscope was used. The Osiris microscope is equipped with an FEI ChemiSTEM detector for high-efficiency EDXS analyses. With this device, twodimensional elemental maps were recorded at high lateral resolution in the STEM mode. The Esprit software (Bruker) was used for quantification of the obtained EDXS data by means of the thin-film approximation.

Atom probe tomography (APT) samples were prepared with the standard lift-out technique using a Zeiss Auriga dual beam Focused Ion beam (FIB). ${ }^{17}$ To be able to measure the whole thin film, an additional Ag layer was sputtered on top prior to the FIB procedure. The APT tip was then prepared to include a fraction of this Ag coating and the whole thin film. The APT measurements were conducted using a Cameca Local Electrode Atom Probe (LEAP 4000X HR) at a set temperature of $25 \mathrm{~K}$, resulting in a tip temperature slightly above $30 \mathrm{~K}$, with a laser pulse energy of $20 \mathrm{pJ}$, a pulse rate of $100 \mathrm{kHz}$ and a detection rate of $0.2 \%$. The datasets were evaluated with Cameca IVAS 3.6.14.

\section{Electrical characterization}

Electrical transport properties were measured on a $70 \mu \mathrm{m}$ wide and $700 \mu \mathrm{m}$ long microbridge structured by laser cutting. The voltage-current characteristics $V(I)$ as well as the temperature dependence of the resistance $R(T)$ were measured in four-point geometry using a $16 \mathrm{~T}$ physical property measurement system over a wide temperature range down to $10 \mathrm{~K}$ and for variable field orientations in the maximum Lorentz force configuration. Further transport data were measured up to the highest possible fields using the $35 \mathrm{~T}$ DC magnet at the National High Magnetic Field Laboratory (NHMFL) in Tallahassee. $T_{\mathrm{c}, 90}$ was determined with a temperature-dependent $90 \%$ criterion of $R_{\mathrm{n}}(T)$. The upper critical field $H_{\mathrm{c} 2}(T)$ was determined with the same $90 \% R_{\mathrm{n}}$ criterion, and the irreversibility field $H_{\text {irr }}$ was determined from resistivity curves using the noise level of $5 \mathrm{~m} \Omega$ as the criterion and from extrapolating $f_{\mathrm{K}}=J_{\mathrm{c}}{ }^{0.5} B^{0.25}$ to zero (Kramer plots ${ }^{\mathbf{1 8 , 1 9}}$ ). These values coincide well at high temperatures. At lower temperatures, an evaluation of $H_{\text {irr }}$ with Kramer plots leads to apparent underestimations of $H_{\text {irr }}$ with large error bars and were therefore omitted. The angular dependence of upper critical field, $H_{\mathrm{c} 2}(\theta)$, where $\theta$ is the angle between the crystallographic $c$-axis and the applied magnetic field, was determined with a field-dependent $90 \%$ criterion of $R_{\mathrm{n}}(B)$. The critical current $J_{\mathrm{c}}$ was determined using an electrical field criterion of $1 \mu \mathrm{V} \mathrm{cm}^{-1}$ and the $N$ value as the slope of the $V(I)$ curves in the double-logarithmic plot. 


\section{Results and discussion}

\section{Structural properties}

Crystallography. X-Ray diffraction in Bragg-Brentano geometry shows $(00 l)$ reflections of the $\mathrm{NdFeAs}\left(\mathrm{O}_{1-x} \mathrm{~F}_{x}\right)$ layer (space group $P 4 / \mathrm{nmm}$ ), the NdOF cap layer, and the $\mathrm{MgO}$ substrate (Fig. 1a), indicating a $c$-axis-orientated film with a lattice parameter $c=0.856 \mathrm{~nm}$ determined with the Nelson-Riley extrapolation. ${ }^{20}$ Additionally, a reflection of FeAs or $\mathrm{FeAs}_{2}$ with very low intensity is visible. The observed $\theta-2 \theta$ scan of the $\mathrm{MgO} /$ $\mathrm{NdFeAs}(\mathrm{O}, \mathrm{F}) / \mathrm{NdOF}$ composite displays a usual quality in texture ( $c$-axis-oriented growth) and crystallinity as expected for thin FBS films.

A reciprocal space map (RSM, Fig. 1b) was analyzed to get information about the in-plane lattice parameter. The scanned range covers the $(113)_{\mathrm{MgO}}$ reflection of the substrate and the $(116)_{\mathrm{NdFeAs}(\mathrm{O}, \mathrm{F})}$ reflection of the superconducting layer. Two (black) maxima at $Q_{x} \approx 0.33$ originate from the $K_{\alpha 1}$ and $K_{\alpha 2}$ of (113) $)_{\mathrm{MgO}}$. For the $(116)_{\mathrm{NdFeAs}(\mathrm{O}, \mathrm{F})}$ reflection, a wide range in $Q_{x}$ and $Q_{z}$ values is visible. A broad variation occurs close to the bulk position at $Q_{x} \approx 0.35$ and $Q_{z} \approx 0.705$ of the $\operatorname{NdFeAs}\left(\mathrm{O}_{1-x} \mathrm{~F}_{x}\right)$ compound.t This peak broadening together
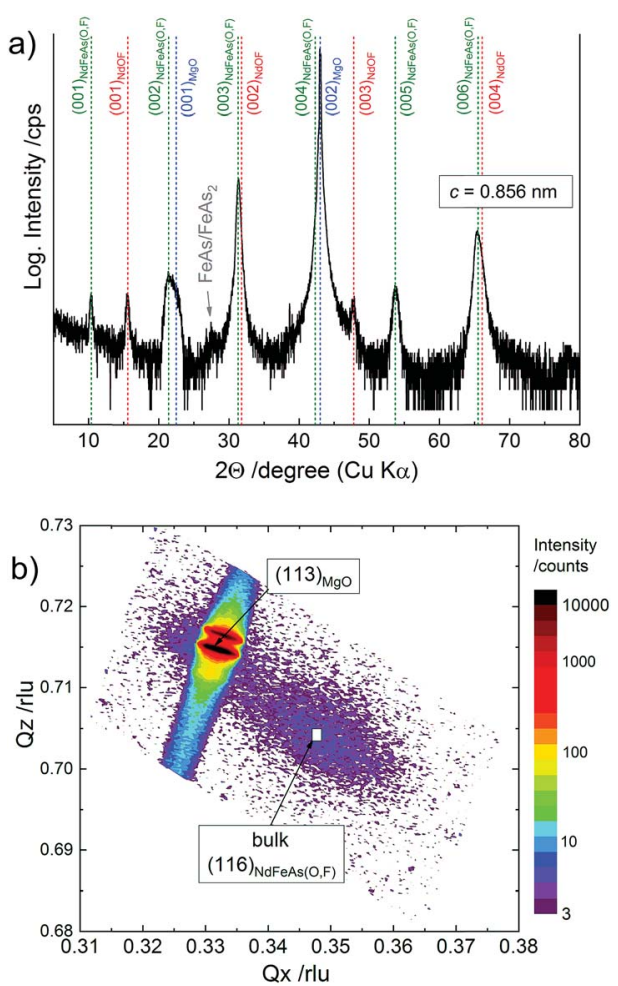

Fig. 1 (a) $\mathrm{X}$-ray $\theta-2 \theta$ scan of the $\mathrm{MgO} / \mathrm{NdFeAs}(\mathrm{O}, \mathrm{F}) / \mathrm{NdOF}$ composite in Bragg-Brentano geometry verifying the $c$-axis-oriented growth of both $\operatorname{NdFeAs}(\mathrm{O}, \mathrm{F})$ and $\mathrm{NdOF}$ by the presence of $(00 l)$-reflections. (b) Reciprocal space map showing a relatively broad (116) reflection of the $\mathrm{NdFeAs}(\mathrm{O}, \mathrm{F})$ layer besides the substrate $(113)_{\mathrm{MgO}}$ reflection.

\$ For modeling of the crystal structure of NdFeAs(O,F), ref. 163839 of the ICSD database was used (see also ref. 8). with a possible variation in the $a$-axis lattice parameter might be caused by several reasons: the formation of local strain at dislocation arrays or around small defects (high dislocation density), the influence of the mosaicity in thin films or a variation of the composition of the $\operatorname{NdFeAs}\left(\mathrm{O}_{1-x} \mathrm{~F}_{x}\right)$ layer. Furthermore, a weak-intensity peak seems to be overlapping with the $(113)_{\mathrm{MgO}}$ reflection which could originate from a partially strained layer at the interface to the substrate. However, from this RSM measurement alone one cannot distinguish between the afore-mentioned strain states.

A high-resolution transmission electron microscopy (HRTEM) image (Fig. 2) indicates a dense $\operatorname{NdFeAs}(\mathrm{O}, \mathrm{F})$ layer with a thickness $t$ of approximately $22 \mathrm{~nm}$. The electron beam, parallel to the $[100]_{\mathrm{NdFeAs}(\mathrm{O}, \mathrm{F})}$ zone axis, reveals the $\operatorname{NdFeAs}(\mathrm{O}, \mathrm{F})$ microstructure. Differences in lattice parameters and epitaxial relations between the various layers are determined by fast Fourier transformation (FFT) patterns of several areas in the different layers. The experimentally obtained diffractograms were compared with selected-area diffraction patterns (not shown here) that were calculated by using the JEMS software. ${ }^{21}$ For the $\operatorname{NdFeAs}(\mathrm{O}, \mathrm{F})$ layer, the diffractogram shows good agreement with the calculated pattern of tetragonal NdFeA$\mathrm{s}(\mathrm{O}, \mathrm{F})$ with the space group $P 4 / n m m$ t along its [100] zone axis. As a general result, the epitaxial relation $(001)[100]_{\mathrm{MgO}} \|(001)$ $[100]_{\mathrm{NdFeAs}(\mathrm{O}, \mathrm{F})} \|(001)[110]_{\mathrm{NdOF}}$ and good crystalline growth are confirmed. Between the $\mathrm{MgO}$ substrate and $\mathrm{NdFeAs}(\mathrm{O}, \mathrm{F})$ layer, however, an additional thin reaction layer is visible $(\leq 2 \mathrm{~nm})$, which was confirmed by energy-dispersive X-ray spectroscopy (EDXS) as magnesium fluoride (see Fig. 3). No grain boundaries are observable in this field of view. In the HRTEM image of Fig. 2, the atomic columns seem to be much better resolved in the NdFO cap layer than in the adjacent $\operatorname{NdFeAs}(\mathrm{O}, \mathrm{F})$ layer. However, the blurred image contrast visible in the latter is probably caused by internal strain, correspondingly lattice distortions, as well as larger crystal defects. The NdOF layer is not continuous and consists of trapezoidally shaped regions (as also observed by atomic force microscopy measurement, see $\mathrm{ESI} \dagger)$.

Microstructure. The elemental distribution in the individual layers was investigated by combined scanning TEM (STEM) and EDXS (Fig. 3). A (discontinuous) reaction layer between the $\mathrm{MgO}$ substrate and $\operatorname{NdFeAs}(\mathrm{O}, \mathrm{F})$ layer (Fig. $3 \mathrm{c}$ and $\mathrm{f}$ ), also seen in TEM with a thickness of up to $2 \mathrm{~nm}$, turned out to be magnesium fluoride and is formed due to the $\mathrm{F}$ diffusion during the deposition. For thermodynamic reasons, presumably the more stable $\mathrm{MgF}_{2}$ phase is present here. Additionally, round as well as slightly in the $c$-axis direction elongated FeAs or $\mathrm{FeAs}_{2}$ particles are observed. These FeAs-rich precipitates are found both in the $\mathrm{NdOF}$ cap layer as well as at the interface between both layers (Fig. 3a and d - brighter Fe \& As, Fig. 3b and e - darker Nd \& O). The EDXS measurements do not allow determining the composition of these FeAs particles exactly. This is particularly hindered by the small size of the particles being embedded in an about $50 \mathrm{~nm}$ thick TEM lamella, yielding a superposition of $\mathrm{X}$-rays excited from all elements within the chosen region under investigation. However, from the image intensity visible in the quantified As map (Fig. 3d) one can assume that the most upper 


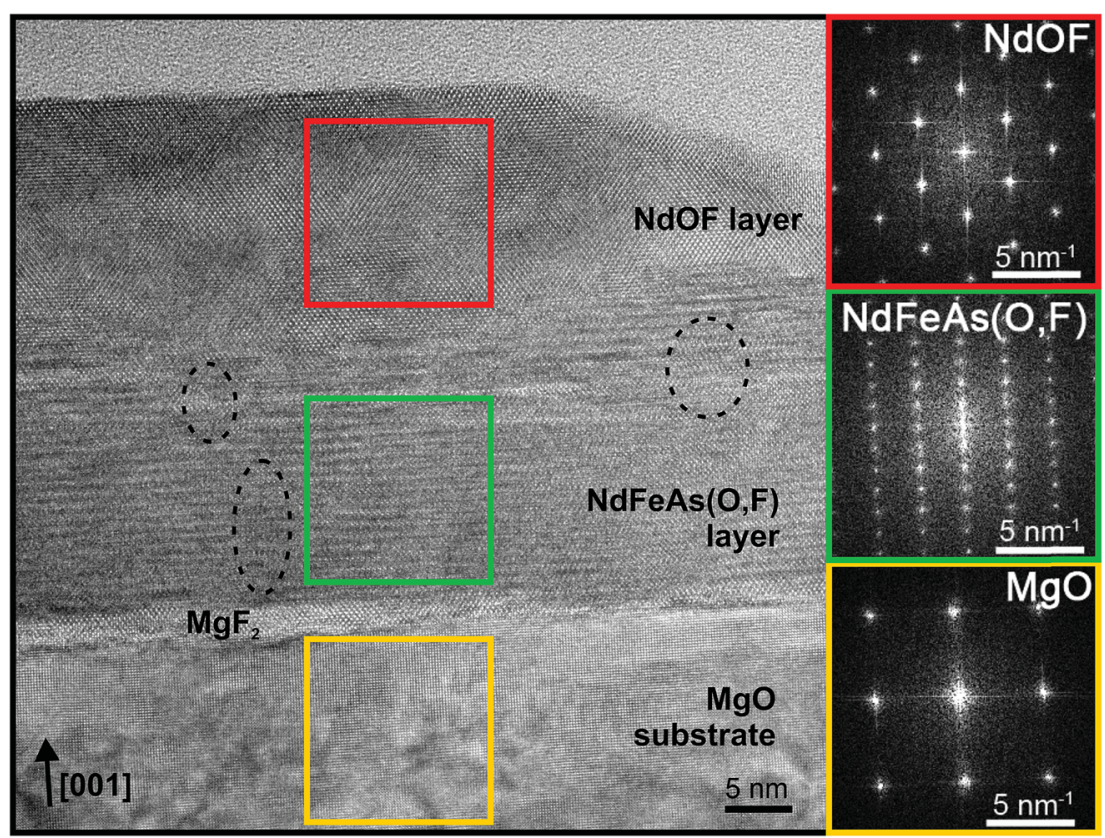

Fig. 2 High-resolution TEM micrograph of the MgO/NdFeAs(O,F)/NdOF sample in cross-sectional view and corresponding FFT patterns obtained from the different layer regions marked by colored squares. An epitaxial growth is confirmed, however, the crystal lattice of the $\operatorname{NdFeAs}(\mathrm{O}, \mathrm{F})$ layer is relatively strongly disturbed.

particles (on top of the NdOF layer) have a higher As content, hinting at $\mathrm{FeAs}_{2}$, whereas the particles embedded within NdOF and close to the interface show a weaker signal intensity as expected for FeAs. Besides these precipitates and the $\mathrm{MgF}_{2}$ reaction layer, F-rich planes with a thickness well below $2 \mathrm{~nm}$ and local inhomogeneities of $\mathrm{Fe}$ and $\mathrm{Nd}$ are visible within the $\operatorname{NdFeAs}(\mathrm{O}, \mathrm{F})$ layer, where occasionally the contents of $\mathrm{Nd}$ and Fe are correlated.

For a closer look at the local element distribution, atom probe tomography (APT) images were recorded on several samples prepared by a focused ion beam (FIB) technique. From the near-atomic-resolution 3D datasets, element distribution maps of the whole FIB samples ( $50 \mathrm{~nm}$ in width) can be created (Fig. 5), both in the cross-section and within the film plane. As clearly visible, the elements are not homogenously distributed within the $\operatorname{NdFeAs}(\mathrm{O}, \mathrm{F})$ film. F-rich and Fe-rich local regions as well as metallic, single-element $\mathrm{Fe}$ nanoparticles of $2 \mathrm{~nm}$ dimension and small NdO precipitates within the superconducting layer are visible. A few FeAs-rich $10 \mathrm{~nm}$ wide, elongated precipitates starting at the interface to the substrate are also visible with APT (for further APT images see ESI†). The inhomogeneous distribution of $\mathrm{O}$ and $\mathrm{F}$ in the cap layer
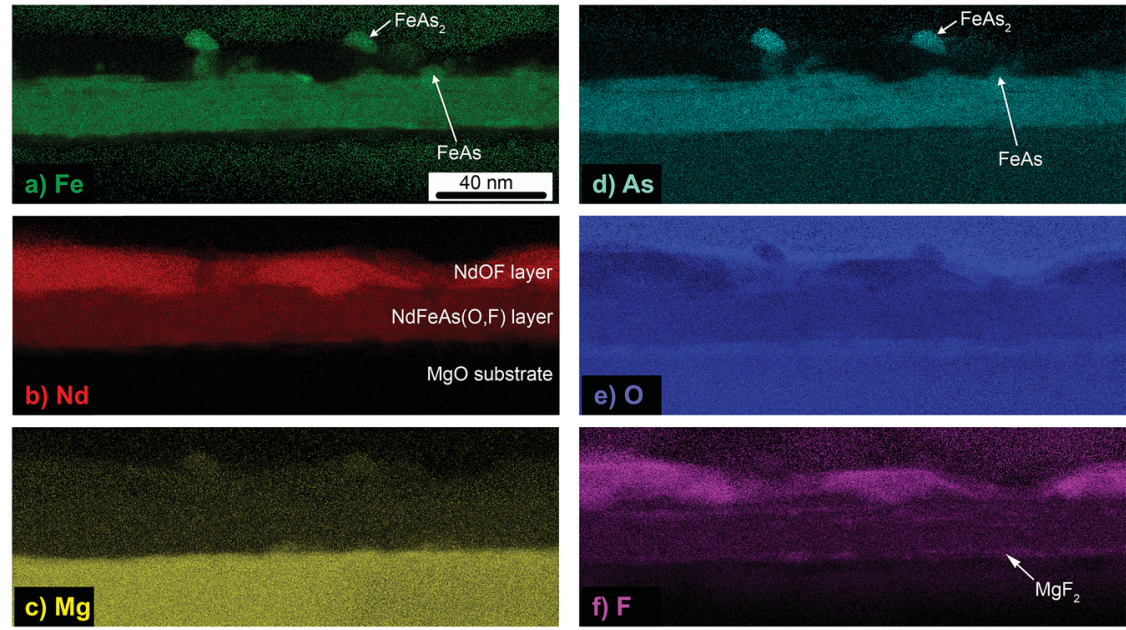

Fig. 3 Element distribution maps of $\mathrm{Fe}(\mathrm{a}), \mathrm{Nd}(\mathrm{b}), \mathrm{Mg}(\mathrm{c}), \mathrm{As}(\mathrm{d}), \mathrm{O}(\mathrm{e})$ and $\mathrm{F}$ ( $\mathrm{f}$ ) of the $\mathrm{NdFeAs}(\mathrm{O}, \mathrm{F}) / \mathrm{NdOF}$ layers on MgO measured by energydispersive $\mathrm{X}$-ray spectroscopy, showing nonstoichiometries on the nanoscale in the $\mathrm{NdFeAs}(\mathrm{O}, \mathrm{F})$ layer as described in the text. FeAs/FeAs 2 precipitates and a reaction layer composed of magnesium fluoride are also observed. 

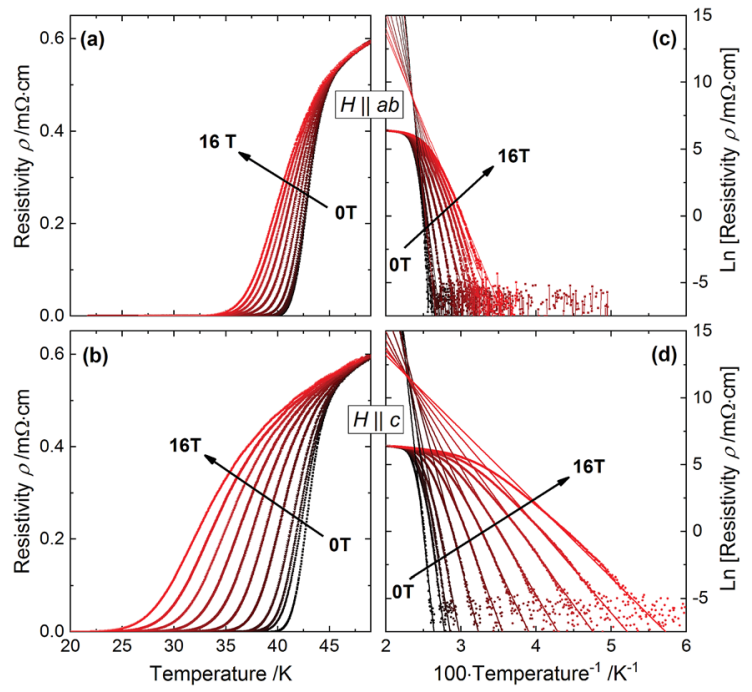

Fig. 4 Resistive transitions $\rho(T)$ measured in static magnetic fields up to $16 \mathrm{~T}$ for both major directions $H \| a b$ (above) and $H \| c$ (below) in linear representation $(a, b)$ as well as in Arrhenius plots $(c, d)$.

originates from the non-stoichiometry of NdOF. Some segregation seems to take place during the deposition process of the NdOF layer: on the one hand F-rich areas and on the other hand areas rich in $\mathrm{FeAs} / \mathrm{FeAs}_{2}$ and $\mathrm{NdO}$ are formed.

This pronounced local inhomogeneity of the elements appears to be in contrast to the uniform layer observed in the high-resolution TEM image (Fig. 2). There, only small distorted areas are barely visible (marked by dashed lines). It seems that the F-rich, Fe-rich and Fe-As local regions are not pronounced defects with a clear atomic structure, or, in the case of the $2 \mathrm{~nm}$ metallic Fe nanoparticles, are too small to observe with XRD (and perhaps TEM). This is clearly different from microstructures of other FBS films, where self-assembled precipitates of clear crystal structure and morphology are observed, for example $4-5 \mathrm{~nm}$ wide nanopillars in epitaxial $\mathrm{BaFe}_{2} \mathrm{As}_{2}$ thin films. $^{22}$

\section{Electrical transport properties}

Superconducting transition, upper critical field, and irreversibility field. To determine the nature of the superconducting transition, the temperature-dependent resistivity, $\rho(T)$, has been measured in static magnetic fields up to $16 \mathrm{~T}$ for both major directions, $H \| c$ and $H \| a b$ (Fig. 4). The superconducting transition $T_{\mathrm{c}, 90}$ defined as $90 \%$ of the temperature-dependent normal state resistivity is $44.7 \mathrm{~K}$ and $T_{\mathrm{c} \text {,onset }}$ defined as the intersection of the linear functions in the transition region and in the normal state (see e.g. ref. 23) is $47.7 \mathrm{~K}$. The small transition width $\Delta T=T_{\mathrm{c}, 90}-$ $T_{\mathrm{c}, 10}$ of $3 \mathrm{~K}$ and the smoothness of the transition suggest that the sample is reasonably homogeneous on the macroscale. With increasing magnetic fields $H \| c$, the superconducting transition of our thin film shows the typical broadening due to thermally activated vortex motion, e.g. a transition temperature of $37 \mathrm{~K}$ with $\Delta T=10 \mathrm{~K}$ at $16 \mathrm{~T}$.

In contrast, the in-field transition temperature of $43.5 \mathrm{~K}$ at $16 \mathrm{~T}$ as well as $\Delta T=6 \mathrm{~K}$ for $H \| a b$ indicates that the $T_{\mathrm{c}}$ for $H \| a b$ is less affected by the magnetic field than for $H \| c$.

The temperature dependencies of the upper critical field, $H_{\mathrm{c} 2}(T)$, and the irreversibility field, $H_{\mathrm{irr}}(T)$, as determined from the resistivity curves $\rho(T)$, and the pinning force density $F_{\mathrm{p}}$ (see Experimental - Electrical characterization), show clear differences for $H \| c$ and $H \| a b$ (Fig. 6a). Because of the lack of low-temperature data, only a very limited range of the magnetic phase diagram is accessible, and it is not possible to reliably fit $H_{\mathrm{c} 2}(T)$ with a suitable model for FBS. To determine the $H_{\mathrm{c} 2}$ anisotropy near $T_{\mathrm{c}}$ as well as the zero-field orbital upper critical fields and coherence lengths, the slope of $H_{\mathrm{c} 2}$ at $T_{\mathrm{c}}$ for both main directions was calculated by linear fits above $2 \mathrm{~T}$ in order to avoid the inhomogeneity-influenced region near $T_{\mathrm{c}}$. These fits meet at $T=45.0 \mathrm{~K}$ which is close to $T_{\mathrm{c}, 90}$. The respective slopes $\mathrm{d} H_{\mathrm{c} 2} / \mathrm{d} T$

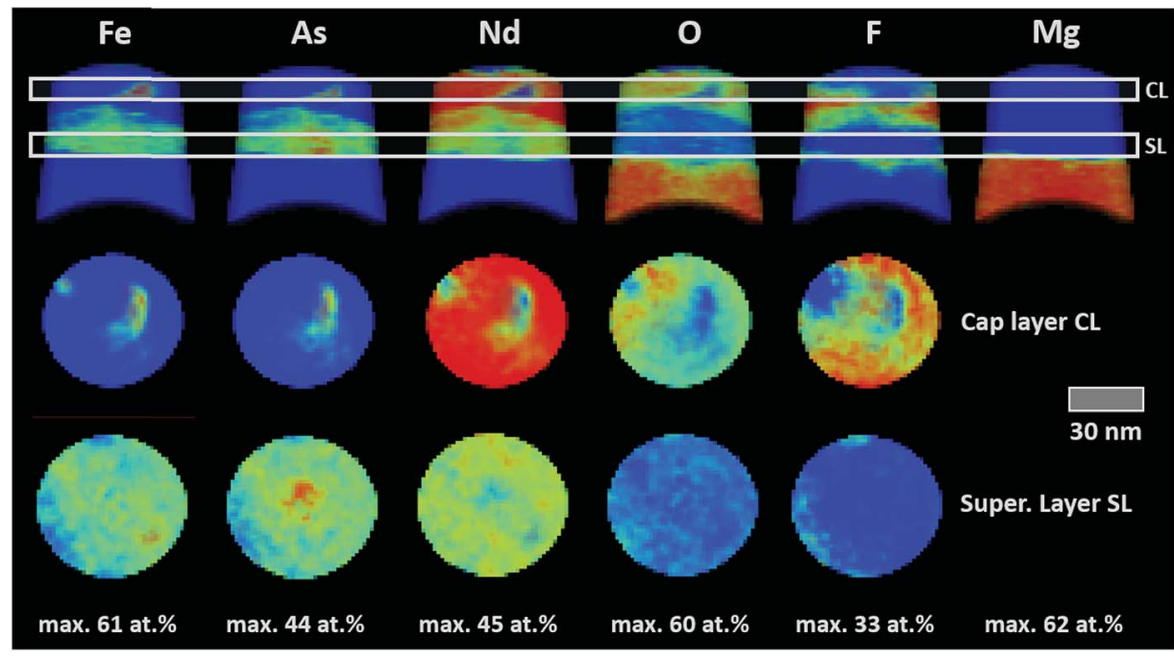

Fig. 5 Color-coded atomic element distribution of $\mathrm{Fe}, \mathrm{Nd}, \mathrm{Mg}, \mathrm{As}, \mathrm{O}$, and $\mathrm{F}$ of a $\mathrm{NdFeAs}(\mathrm{O}, \mathrm{F}) / \mathrm{NdOF}$ layer on MgO measured by APT, in the vertical cross-section (above), in-plane cut of the cap-layer (middle), and in-plane cut of the superconducting layer (below). Blue means 0 at\% and red the corresponding maximum concentration. Small Fe, Fe-As and $\mathrm{NdO}$ particles are visible. 


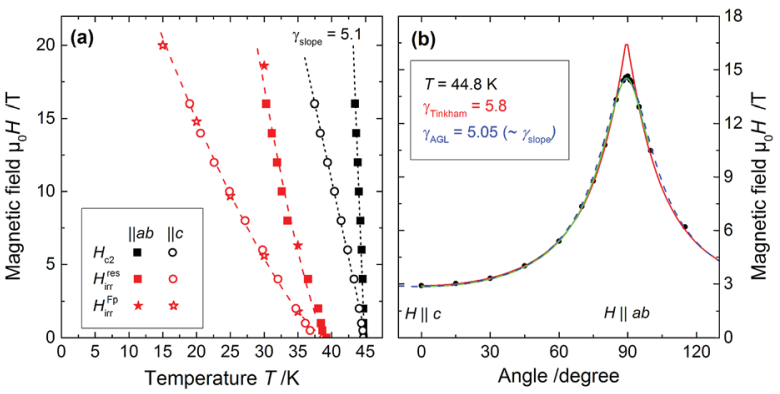

Fig. 6 Temperature dependence of the upper critical field, $\mu_{0} H_{\mathrm{c} 2}$, and irreversibility field, $\mu_{0} H_{\text {irr, }}$ for both major directions. The dashed and dotted curves are power law fits $H_{\text {irr }}(T)=H_{\text {irr }}(0)\left(1-T / T_{\text {irr }}\right)^{a}$ and linear fits to $H_{\mathrm{c} 2}$ above $2 \mathrm{~T}$ respectively. (b) Angular dependence of the upper critical field at $44.8 \mathrm{~K}$, i.e. close to $T_{c \text {,onset. }}$ The solid line (red) is a fit using the 2D Tinkham model, the dashed line (blue) represents the one-band anisotropic Ginzburg-Landau (AGL) theory, and the green line the results for the modified AGL model (see text).

for $H \| c$ and $H \| a b$ are $-2.1 \mathrm{~T} \mathrm{~K}^{-1}$ and $-11.2 \mathrm{~T} \mathrm{~K}^{-1}$ resulting in an $H_{\mathrm{c} 2}$ anisotropy of $\gamma_{H_{\mathrm{c} 2}}=5.1 \pm 0.3$ at $T_{\mathrm{c}}$. This value is in good agreement with other 1111 compounds. ${ }^{13,24,25}$ The (single-band) orbital upper critical fields according to the $\mathrm{WHH}$ model $^{\mathbf{1}}$ are $65 \pm 1 \mathrm{~T}$ and $330 \pm 7 \mathrm{~T}$ for $H \| c$ and $H \| a b$ respectively, and the corresponding coherence lengths are $\xi_{c}=0.44 \pm 0.02 \mathrm{~nm}$ and $\xi_{a b}=2.25 \pm 0.02 \mathrm{~nm}$. The irreversibility field for both directions could be fitted with the power law $H_{\mathrm{irr}}(T)=H_{\mathrm{irr}}(0)\left(1-T / T_{\mathrm{irr}}\right)^{a}$. Best fits were achieved with $T_{\mathrm{irr}}=39.2 \mathrm{~K}, a=1.38$, and $H_{\mathrm{irr}}(0)$ values of $37 \mathrm{~T}$ and $125 \mathrm{~T}$ for $H \| c$ and $H \| a b$, respectively.

The sample's anisotropy near $T_{\mathrm{c}}$ was further evaluated by measuring the angular dependence of the upper critical field, $H_{\mathrm{c} 2}(\theta)$, where $\theta$ is the angle between the applied field and the $c$ axis. $H_{\mathrm{c} 2}(\theta)$ was determined from $R(B)$ curves at given angles at $44.8 \mathrm{~K}$. Since the $R(T)$ and the $R(B)$ measurements are separated by half a year, the sample altered slightly (increased normal state resistivity likely due to undesired moisture exposure); $T_{\mathrm{c}, \text { onset }}$, however, was unchanged. $H_{\mathrm{c} 2}(\theta)$, Fig. 6b, shows as expected a maximum at $\theta=90^{\circ}(H \| a b)$ and a minimum at $\theta=0^{\circ}(H \| c)$. The data can be described fairly well using the 3D Anisotropic Ginzburg-Landau (AGL) model ${ }^{26,27}$ with $H_{\mathrm{c} 2}(\theta)=H_{\mathrm{c} 2}{ }^{c}\left(\cos ^{2} \theta+\gamma^{-2} \sin ^{2} \theta\right)^{-0.5}$, blue line in Fig. 6b. However, even though the fit describes the data nicely near $H \| c$ and $H \| a b$, it clearly deviates from the data at medium angles.

There are two possible reasons for this behavior: firstly, different extended defects as observed in TEM (Fig. 3) and APT (Fig. 5) may lead to extra contributions near the two major directions and consequently alter the anisotropy of the coherence lengths. This effect has been described for columnar defects in irradiated Sm1111 single crystals ${ }^{28}$ as well as $a b$-planar defects in CaAlSi single crystals ${ }^{29}$ and has been discussed for Co-doped ${ }^{30}$ and P-doped ${ }^{31} \mathrm{BaFe}_{2} \mathrm{As}_{2}$ (Ba122) thin films with several different growth defects. In fact, following the modified AGL model in those references, which has recently been generalized by Talantsev and Mataira, ${ }^{32}$ and decreasing the exponent in the above equation to 1.85 lead to a near-perfect fit (green curve in Fig. 6b). Secondly, recently a mixture of 2D and 3D fluctuations has been described by Galeski et al. on Sm1111 nanocrystals which may extend the temperature region of low-temperature $2 \mathrm{D}$ behavior (below the expected dimensional cross-over temperature $T_{\text {co }}=T_{\mathrm{c}}\left(1-\left(\xi_{c} / d\right)^{2}\right)=35 \mathrm{~K}$ with $\left.d=0.8 \mathrm{~nm}\right)$ close to $T_{\mathrm{c}}$ since 2D fluctuations may screen the 3D ones. ${ }^{33}$ Indeed, a fit according to the Tinkham model ${ }^{34}$ for 2D superconductors $\left|H_{\mathrm{c} 2}(\theta) \sin \theta\right|$ $H_{\mathrm{c} 2}{ }^{c}|+| H_{\mathrm{c} 2}(\theta) \cos \theta /\left.H_{\mathrm{c} 2}{ }^{a b}\right|^{2}=1$ (we used it in the form of eqn (3) of ref. 35) to the low- and medium-angle data with $H_{\mathrm{c} 2} \| c$ as the fixed parameter (red line in Fig. 6b) results in a very good agreement with the data up to $85^{\circ}$ for $\gamma_{\text {Tink }}=5.8$. This value, however, is slightly higher than $\gamma_{H_{\mathrm{c} 2}}=5.1 \pm 0.3$. A very similar mix of 2D and 3D $H_{\mathrm{c} 2}$ anisotropy behavior near $T_{\mathrm{c}}$ had been found for $\mathrm{Bi}_{2} \mathrm{Sr}_{2} \mathrm{CaCu}_{2} \mathrm{O}_{x}$ single crystals by Marcon et al. ${ }^{36}$ To determine whether one of these two effects and which one might be responsible for the observed $H_{\mathrm{c} 2}$ anisotropy would need further investigations.

Pinning potential. The activation energy $U_{0}$ for flux motion (Fig. 7) is estimated by linear fits $\ln \rho(T, H)=\ln \rho_{0}(H)-U_{0}(H) / k_{\mathrm{B}} T$ to the relevant region in the respective Arrhenius plots (Fig. $4 \mathrm{~b}$ and d) with field-independent $\rho\left(T_{\mathrm{c}}\right)$, where $\rho_{0}$ is a prefactor and $k_{\mathrm{B}}$ the Boltzmann constant. The field dependence of $U_{0}$ for $H \| c$ is well described by the function $U_{0} \propto H^{-\alpha}\left(1-H / H^{*}\right)^{\beta}$ which was first proposed for $\mathrm{MgB}_{2}$ by Thompson et $a l^{37}$ who mentioned that the exponents should be similar to the ones in the field dependence of the pinning force density since $U_{0}$ and $J_{\mathrm{c}}$ are inherently related.

Due to the lack of high-field data above $16 \mathrm{~T}$, the exponents as well as $H^{*}$, which is close to $H_{\text {irr }}(0 \mathrm{~K})$, cannot be determined with absolute certainty. Nevertheless, setting $\alpha=p-1$ with $p \sim 0.7$ as determined in $F_{\mathrm{p}}$ scaling (see below), good agreement with the data was achieved with, e.g., $\alpha=0.3, \beta=5$ and $H^{*}=70 \mathrm{~T}$. These values are reasonable, whereas $\alpha=0.5$ and $\beta=2$ as often used and leading to similarly good fits yield an unrealistically low $H^{*}$ of $45 \mathrm{~T}$. For $H \| a b$ the lack of high-field data is even more problematic and the second factor in the above equation cannot be determined at all. Nevertheless, above $3 \mathrm{~T} U_{0}$ follows a power law with $\alpha=0.7$ for that direction. At lower fields, the data differ from these functions because of the crossover to the regime of single vortex pinning, where $U_{0}$ is constant. The value of this cross-over field to single vortex pinning of $\sim 2-3 \mathrm{~T}$ is comparable to literature data on Nd1111, La1111, and Sm1111 (ref. 38 and references therein).

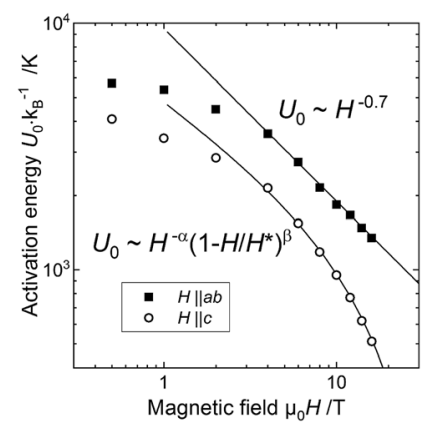

Fig. 7 Field dependence of the pinning potential for both major directions. The solid lines are fits to the equations, see text. 
Critical current density. Field and angular dependence of the critical current density $J_{\mathrm{c}}$ was measured up to $35 \mathrm{~T}$ at $4.2 \mathrm{~K}$ and up to $16 \mathrm{~T}$ at further temperatures up to $30 \mathrm{~K}$. The field dependence of $J_{\mathrm{c}}(H)$ was measured for both major directions ( $H \| c$ and $H \| a b$, Fig. 8). $J_{\mathrm{c}}$ for $H \| c$ is always lower than for $H \| a b$, which is a consequence of the $H_{\mathrm{c} 2}$ and hence $H_{\text {irr }}$ anisotropy. In the $22 \mathrm{~nm}$ film the self-field (s.f.) critical current density $J_{\mathrm{c}, \mathrm{sf}}$ is $7.6 \mathrm{MA} \mathrm{cm}^{-2}$ at $4.2 \mathrm{~K}$ and $J_{\mathrm{c}, \mathrm{sf}}=2.2 \mathrm{MA} \mathrm{cm}{ }^{-2}$ at $20 \mathrm{~K}$. With increasing field, $J_{\mathrm{c}}$ monotonously decreases. At high magnetic fields of $35 \mathrm{~T}, J_{\mathrm{c}}(4.2 \mathrm{~K})$ still reaches very high values of $2.8 \mathrm{MA}$ $\mathrm{cm}^{-2}$ and $70 \mathrm{kA} \mathrm{cm}^{-2}$ for $H \| a b$ and $H \| c$, respectively. The weak field dependence for $H \| a b$ at $4.2 \mathrm{~K}$ suggests the presence of intrinsic pinning originating from the modulation of the superconducting order parameter along the $c$-axis as described in detail in ref. 12 as well as possible pinning at the F-rich $a b$ planar defects observed in the F distribution obtained by STEM/ EDXS (Fig. 3f) and APT (Fig. 5).

Compared to earlier investigations on other 1111 compounds $^{11,12}$ (Fig. 8c and d), $J_{\mathrm{c}, \mathrm{sf}}$ is more than doubled. Only for $H \| c$ and fields above $25 \mathrm{~T}$ does the Sm1111 take over. Other FBS like Co-doped Ba122 exhibit a lower self-field $J_{\mathrm{c}, \mathrm{sf}}$. For example a $350 \mathrm{~nm}$ thick $\mathrm{Ba}\left(\mathrm{Fe}_{1-x} \mathrm{Co}_{x}\right)_{2} \mathrm{As}_{2}$ film ${ }^{39}$ exhibits $J_{\mathrm{c}, \mathrm{sf}} \sim$ $5.6 \mathrm{MA} \mathrm{cm}^{-2}$ at $4.2 \mathrm{~K}$ and $J_{\mathrm{c}}$ for $H \| a b$ of $0.18 \mathrm{MA} \mathrm{cm}^{-2}$ at $35 \mathrm{~T}$ (Fig. 8d). $\mathrm{A} \mathrm{BaFe}_{2}(\mathrm{As}, \mathrm{P})_{2}$ film ${ }^{23}$ shows a $J_{\mathrm{c}, \mathrm{sf}}$ of up to $6.5 \mathrm{MA} \mathrm{cm}^{-2}$ at $4.2 \mathrm{~K}$ and a $J_{\mathrm{c}}$ for $H \| a b$ of $0.1 \mathrm{MA} \mathrm{cm}^{-2}$ at $30 \mathrm{~T}$ (not shown here). This shows that the decrease in $J_{\mathrm{c}}$ with increasing field is much more pronounced in Ba122 compared to 1111 compounds. Another study on P-doped Ba122 (ref. 40) showed a $J_{\mathrm{c}, \mathrm{sf}}$ of $12 \mathrm{MA} \mathrm{cm} \mathrm{cm}^{-2}$ by magnetization and $7 \mathrm{MA} \mathrm{cm}^{-2}$ by transport, but the field dependence was not measured. The reason for the high $J_{\mathrm{c}}$ is not clear; the authors supposed the formation of Fe nanoparticles or layers, which however could not be observed with the applied X-ray diffraction. The reasons

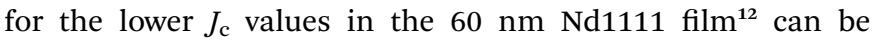
manifold: a different microstructure (e.g. F-content, precipitates), less strain effect (as deduced from a different F-content series, not shown here) and larger thickness itself. ${ }^{41}$

The angular dependencies of the critical current density $J_{\mathrm{c}}(\theta)$ (Fig. 9) show for all fields and temperatures one maximum at $H \| a b\left(\theta=90^{\circ}\right)$, which becomes sharper with increasing field or temperature. No second local maximum at $H \| c$ due to correlated defects arises. The absence of this peak is in agreement with the TEM observations, where no nanocolumns are visible (Fig. 2). Point defects as well as the randomly distributed small precipitates of different compositions seem to act as more or less isotropic pinning and lead to a general increase in $J_{\mathrm{c}}$ over the complete angular range.

To identify pinning contributions originating from these random uncorrelated (isotropic) defects, the $J_{\mathrm{c}}(\theta)$ curves were analyzed using the AGL scaling approach introduced by Blatter et $a .^{42,43}$ This scaling approach was developed for single-band superconductors but has also been successfully applied to multiband materials of FBS like Co-doped Ba112 (ref. 44) and La1111. ${ }^{13}$ The $J_{\mathrm{c}}$ data are scalable in an effective magnetic field $H_{\text {eff }}=H \sqrt{\cos ^{2} \theta+\gamma_{J}^{-2} \sin ^{2} \theta}$. To rescale the data onto a single trend line (Fig. 8e), the $J_{\mathrm{c}}$ anisotropy parameter $\gamma_{J}$ has to be increased with decreasing temperature. It ranges from 1.5 at

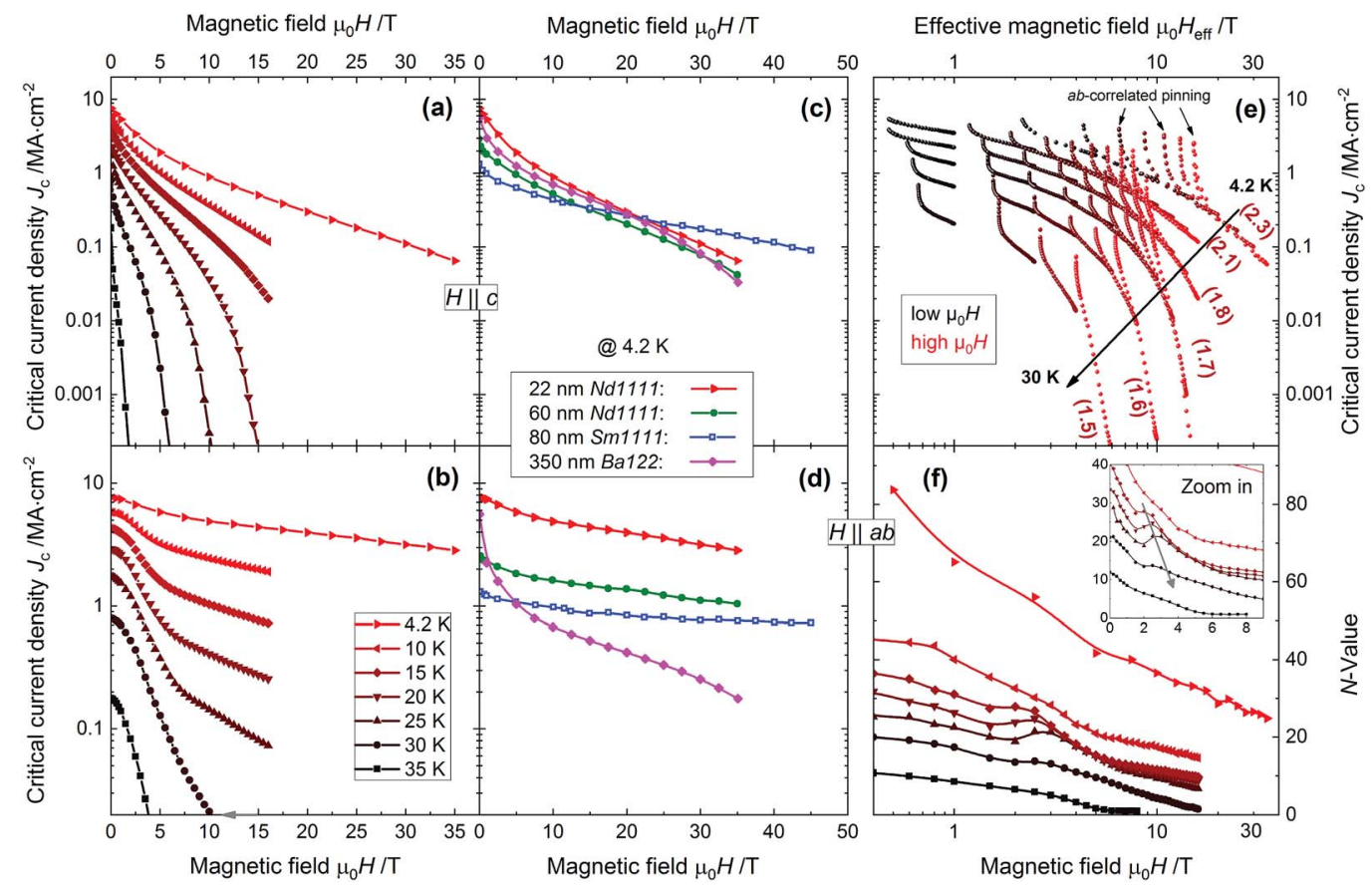

Fig. 8 Field dependence of the critical current density for both major directions $H \| c$ (above) and $H \| a b$ (below) at various temperatures between $4.2 \mathrm{~K}$ and $35 \mathrm{~K}(\mathrm{a}, \mathrm{b})$, and in comparison to data for other 1111 and Co-doped Ba122 from ref. 11, 12, and 39 at $4.2 \mathrm{~K}$ (c, d). Anisotropic GinzburgLandau scaling $(\mathrm{e})$ of the angular dependence $J_{c}(\theta)$ with the used anisotropy parameter $\gamma_{J}$ written in brackets. The curves were measured up to 16 T between $35 \mathrm{~K}$ and $10 \mathrm{~K}$ and up to $35 \mathrm{~T}$ at $4.2 \mathrm{~K}$. Deviations from scaling occur due to $a b$-correlated pinning. (f) $\mathrm{N}$-value at various temperatures between $4.2 \mathrm{~K}$ and $35 \mathrm{~K}$. Inset: zoom in close to the matching field (arrow) observed between $2.4 \mathrm{~T}$ and $3 \mathrm{~T}$ (depending on temperature). 

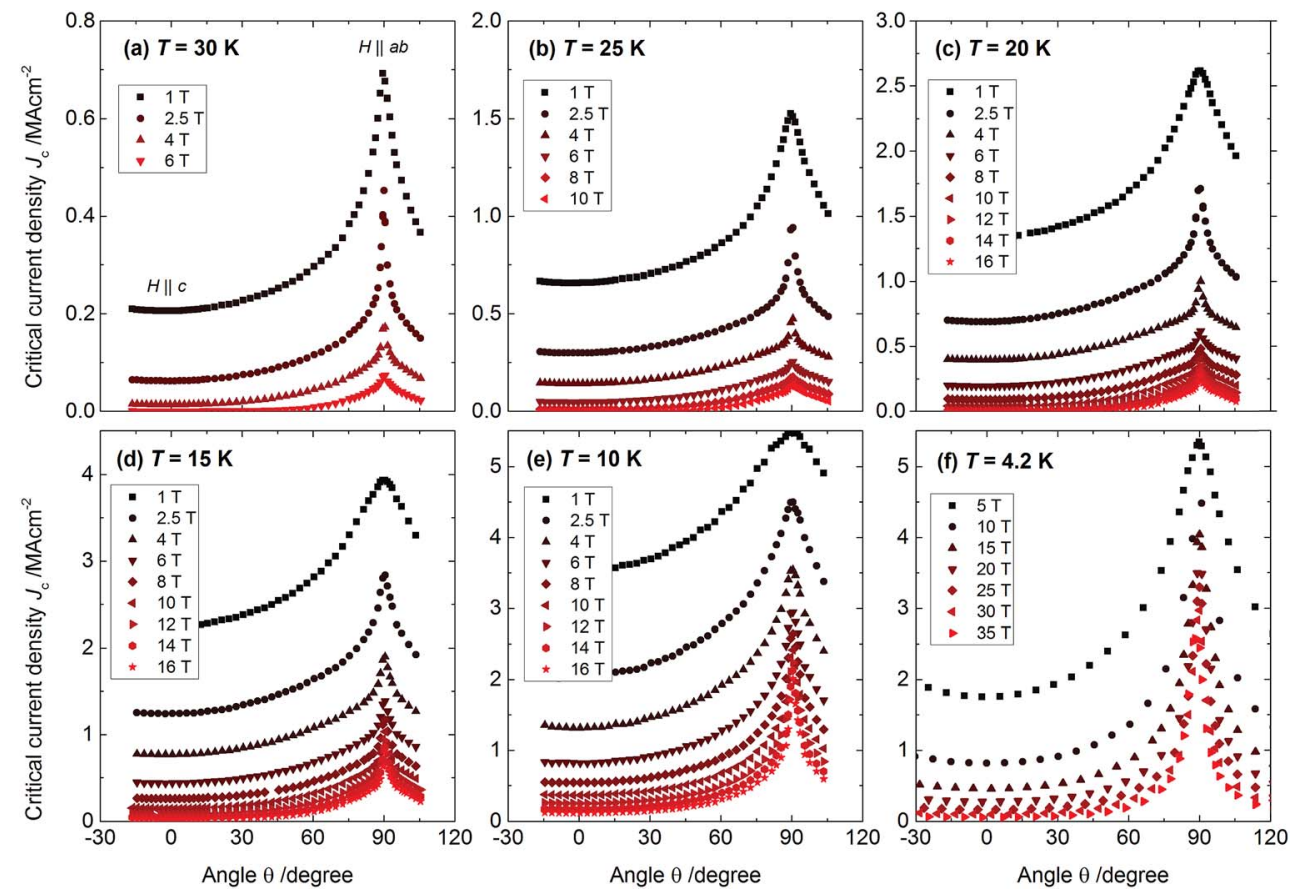

Fig. 9 Angular dependence of the critical current density $J_{c}$ at (a) $30 \mathrm{~K}$, (b) $25 \mathrm{~K}$, (c) $20 \mathrm{~K}$, (d) $15 \mathrm{~K}$, (e) $10 \mathrm{~K}$ and (f) $4.2 \mathrm{~K}$ for various magnetic fields.

$30 \mathrm{~K}$ to 2.3 at $4.2 \mathrm{~K}$. A temperature-dependent anisotropy is typical for multi-band superconductors ${ }^{45}$ and was verified for FBS $^{\mathbf{1 2 , 1 3 , 4 4 , 4 6 , 4 7}}$ as different bands contribute to $J_{\mathrm{c}}$ at different temperatures. However, different trends of the temperature dependence of $\gamma_{J}$ have been reported in the literature depending on the compound and dimensionality. ${ }^{\mathbf{1 2 , 1 3 , 4 7}}$ Up to now, the reason for those differences in temperature dependence is not completely understood, nevertheless decreasing $\gamma_{J}$ values for decreasing temperatures are often found for 3D FBS materials such as Ba122 compounds and increasing values for $2 \mathrm{D}$ compounds. Furthermore, the anisotropy $\gamma_{J}$ of the $22 \mathrm{~nm}$ sample (1.5-2.3) is lower than $\gamma_{H_{\mathrm{c} 2}}=5.1$. This is, generally, due to the influence of extended and correlated defects.

For FBS, the $\xi$ and $\lambda$ anisotropies are different (i.e. $\xi_{a b} / \xi_{c} \neq \lambda_{\mathrm{c}}$ ) $\left.\lambda_{\mathrm{ab}}\right)$ due to the multi-band state and the crossover between $2 \mathrm{D}$ and 3D vortex behavior (see, e.g. ref. 12). In our films, the increase of the $J_{\mathrm{c}}$ anisotropy $\gamma_{J}$ with decreasing temperature is similar to the penetration depth anisotropy $\gamma_{\lambda} ;{ }^{12}$ only the magnitude of $\gamma_{J}$ is smaller than that of $\gamma_{\lambda}{ }^{46}$ This indicates that both intrinsic and extrinsic parameters and also the crossover between $2 \mathrm{D}$ and $3 \mathrm{D}$ behavior affect $J_{\mathrm{c} .}{ }^{12}$

The scaling of $J_{\mathrm{c}}$ on $H_{\text {eff }}$ also exposes possible $a b$-planar and $c$-axis-correlated pinning through deviations from the envelope function. In our films, such deviations occur only at $H \| a b$, due to $a b$-correlated pinning, which is more pronounced at higher fields (examples are marked by black arrows in Fig. 8e) at low as well as higher temperatures. A strong and pronounced deviation for $H \| a b$ at higher temperatures could be an indication for vortex interactions with the surface since the thickness-topenetration depth ratio $(t / \lambda)$ decreases at higher temperatures. On the other hand, no hint for $c$-axis-correlated pinning (i.e. deviation from scaling) is visible.
The $I-V$ curves, from which $J_{\mathrm{c}}$ was determined, are described by the power-law relation $V \sim I^{N}$ around $J_{\mathrm{c}}$ in the entire field and temperature range. The $N$ value (Fig. $8 \mathrm{f}$ ) is in general influenced by sample homogeneity and flux creep rate $S,{ }^{48-51}$ where the former prevails in low- $T_{\mathrm{c}}$ superconductors and the latter in cuprates and FBS with relatively large fluctuation regimes, i.e. Ginzburg numbers Gi. Neglecting inhomogeneities, the creep rate $S$ can be approximated as $S=1 /(N-1) . N$ (as well as $J_{\text {c }}$ ) is usually proportional (or positively correlated) to the pinning potential $U$. Therefore, in general a power-law relation of the type $N \sim J_{\mathrm{c}}{ }^{z}$ has been found e.g. for $\mathrm{YBa}_{2} \mathrm{Cu}_{3} \mathrm{O}_{7}$ (ref. 52) and Nd1111 (ref. 12) in the case of random isotropic pinning, independent of temperature, field and angle. This correlation has nevertheless also been reported for inhomogeneity-driven superconductors, e.g. for $\mathrm{MgB}_{2}, \mathrm{NbTi}$, and $\mathrm{Nb}_{3} \mathrm{Sn}^{.53-55}$ Accordingly, an increase of $J_{\mathrm{c}}$ leads to an increase in the $N$ value, and as a consequence $J_{\mathrm{c}}(H)$ and $N(H)$ should have similar trends. For $H \| a b$, the $22 \mathrm{~nm} \operatorname{NdFeAs}(\mathrm{O}, \mathrm{F})$ film (Fig. 8b and f) shows this trend at low $(4.2 \mathrm{~K})$ and high temperatures $(35 \mathrm{~K})$. Between 15 and $25 \mathrm{~K}$, however, a small maximum in $N(H)$ is formed at around 2.4 T. Such regions of inverse correlation between $J_{\mathrm{c}}$ and $N$ are usually hints for further contributions to the creep rate such as formation of vortex kinks and double kink structures at highly correlated pinning centers leading to increased creep rates if not suppressed by matching densities of vortices and defects, which is called the matching-field effect as discussed later in the paper.

Pinning force density. The field dependence of the pinning force density $F_{\mathrm{p}}(H)$, Fig. 10, depends strongly on temperature and field orientation and reaches a maximum of $F_{\mathrm{p}}(4.2 \mathrm{~K})=$ 95.5 $\mathrm{GN} \mathrm{m}^{-3}$ at $5 \mathrm{~T}$ for $H \| c$, whereas for $H \| a b$ the maximum exceeds $F_{\mathrm{p}}(4.2 \mathrm{~K}, 35 \mathrm{~T})=992.5 \mathrm{GN} \mathrm{m}^{-3}$. The maximum $F_{\mathrm{p}}$ is not 


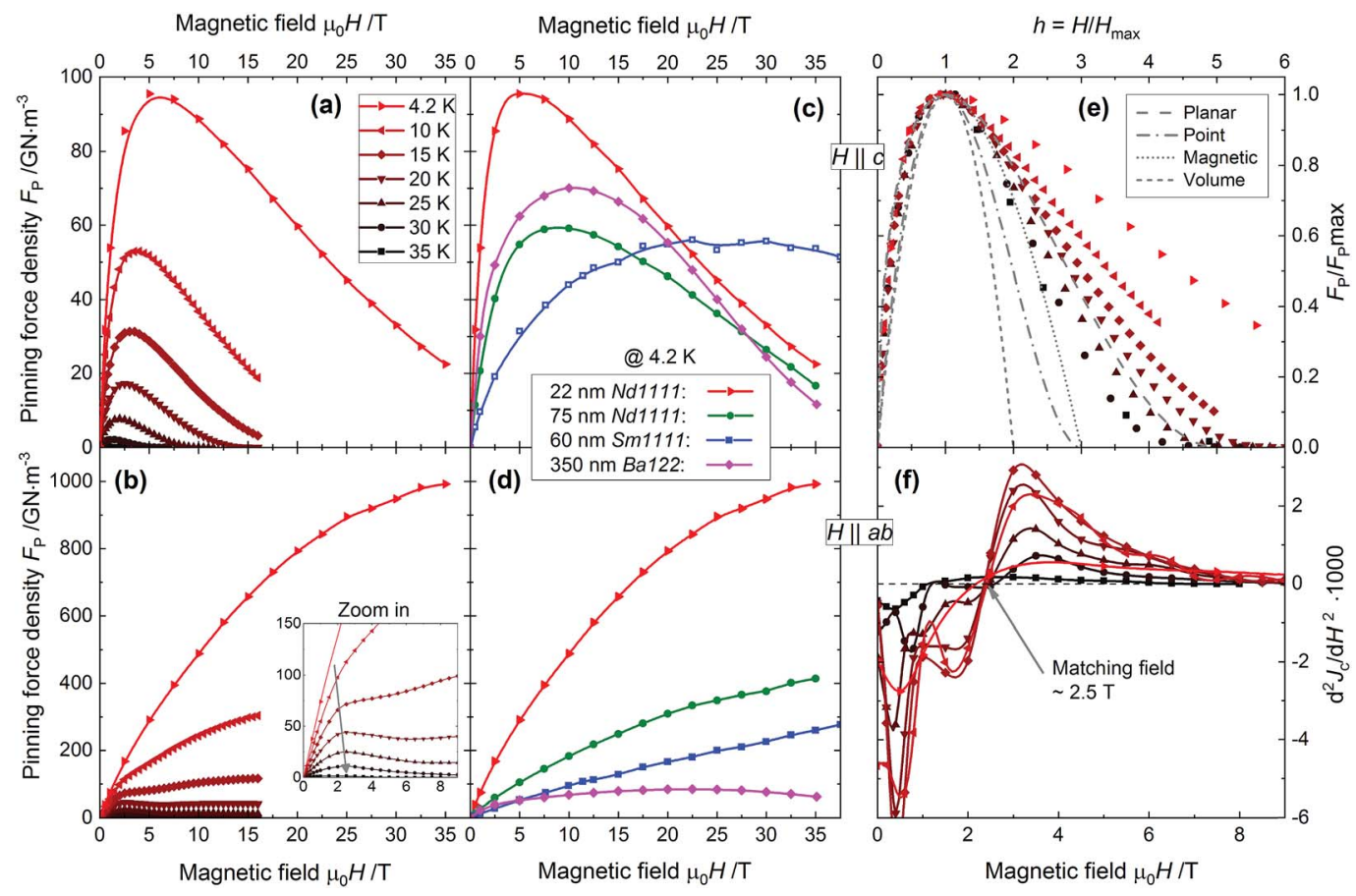

Fig. 10 Field dependence of flux pinning density $F_{\mathrm{p}}$ for both major directions $H \| c$ (above) and $H \|$ ab (below) for various temperatures between $4.2 \mathrm{~K}$ and $35 \mathrm{~K}$ ( $\mathrm{a}$ and $\mathrm{b}$ ), and in comparison to data for other Ln1111 compounds from ref. 11, 33, and 34 at $4.2 \mathrm{~K}$ (c and d). The normalized pinning force density as a function of reduced field $h(\mathrm{e})$. The different lines represent different pinning mechanisms (point, planar, magnetic and volume pinning), where the pinning is dominated by planar defects. (f) Second derivative of $J_{c}(H)$ showing the matching field ( 2.4 T) (see also inset (b)) for different temperatures by crossing the zero-line.

reached for $H \| a b$ at $35 \mathrm{~T}$, indicating that it potentially exceeds $1 \mathrm{TN} \mathrm{m}^{-3}$. These are the highest $F_{\mathrm{p}}$ values ever reported for FBS (Fig. 10c and d), cf. ref. 11, 12 and 39.

Usually, the normalized pinning force density $f_{\mathrm{p}}=F_{\mathrm{p}} / F_{\mathrm{p} \text {,max }}$ is plotted as a function of reduced field $h=H / H_{\text {irr }}$ for superconductors with large vortex liquid regions such as cuprates and FBS. Instead, we plot $f_{\mathrm{p}}$ for $H \| c$ as a function of $h^{*}=H / H_{\max }$ (where $H_{\max }=H\left(F_{\mathrm{p} \text {,max }}\right)$ ) since $J_{\mathrm{c}}$ could not be measured up to $H_{\text {irr }}$ at all temperatures (Fig. 10e). Similar scaling has been used earlier also for $\mathrm{YBa}_{2} \mathrm{Cu}_{3} \mathrm{O}_{7}$ (ref. 56) and FBS. ${ }^{31}$ For $H \| c, F_{\mathrm{p}}(H)$ is analyzed using the modified Dew-Hughes model, where the original scaling field $H_{\mathrm{c} 2}$ is replaced by $H_{\mathrm{irr}} \cdot f_{\mathrm{p}} \propto h^{p}(1-h)^{q} \propto$ $h^{* p}\left(\{p+q\} / p-h^{*}\right)^{q}$ where $p$ and $q$ are free parameters, dependent on the pinning mechanism. ${ }^{57}$ For $H \| a b$, we could not follow this approach because the maximum $F_{\mathrm{p}}$ is not reached.

Attempts to fit $f_{\mathrm{p}}(h)$ or $f_{\mathrm{p}}\left(h^{*}\right)$ with a single such Dew-Hughes function were not successful, especially at low $T$, which suggests that more than one pinning mechanism is present. Nevertheless, the normalized $f_{\mathrm{p}}(H \| c)$ curves scale roughly onto one master curve below $h^{*}<1$ (Fig. 10e), indicating that the same pinning mechanism contributes to $J_{\mathrm{c}}$ at all temperatures in this field range. The exponent $p$ is around 0.7 for all temperatures for $H \| c$. According to ref. 56, that suggests strong pinning centers slightly larger than $\xi_{a b}$, which is in accord with the nanoparticle precipitation seen in TEM and APT. In the highfield range, $f_{\mathrm{p}}$ varies systematically with temperature and field strength. Therefore, specific functions with different $p$ and $q$ parameters ${ }^{57}$ are presented in Fig. 10e: (1) a parameter set of $(p, q)=(0.5,1)$ describes magnetic pinning, $(2)(p, q)=(1,1)$ volume pinning, $(3)(p, q)=(0.5,2)$ planar pinning on twodimensional non-magnetic defects and $(4)(p, q)=(1,2)$ point pinning on non-superconducting particles. ${ }^{57}$ Below $h<1$ the data at all temperatures are closer to both magnetic and planar pinning with $(p, q)=(0.5,1)$ and $(0.5,2)$. Since switching of magnetic domains happens below $200 \mathrm{mT}$ and magnetic pinning is dominated by the penetration depth $\lambda$, magnetic pinning (if at all) only contributes at very low fields. For higher fields, planar pinning on two-dimensional defects dominates such as small-angle grain boundaries or dislocation networks although they have not been directly confirmed in the TEM investigations. Also the model proposed by Kramer for flux shearing on arrays of $1 \mathrm{D}$ defects generates a similar functional dependence to planar pinning. ${ }^{58}$ Another type of magnetic pinning, different from the mechanism described by DewHughes might be possible in these films. In case the elemental Fe nanoparticles are not superparamagnetic, they might contribute magnetically to the pinning force via a reduction of Lorentz force by the additional magnetic flux as described by Blamire et al. ${ }^{59}$ In that case, it would not be limited to the smallest fields.

In the field dependence of $F_{\mathrm{p}}$ (Fig. 10b, inset) and of the $N$ value for $H \| a b$ (Fig. 8f), peaks at around $2.5 \mathrm{~T}$ are found. This peak effect is further evidenced by zero transition of the second derivative of $J_{\mathrm{c}}(H)$ (Fig. 10f). The matching field $B_{0}$ for $H \| a b$ is calculated as $B_{0}=\Phi_{0} / \sqrt{3} \gamma l^{2}$, where $l$ is the vortex row separation in the $c$-direction and $\gamma$ the relevant anisotropy. If $l$ would be equal to the $c$-axis lattice parameter of $0.856 \mathrm{~nm}$, the 
matching field would amount to $\sim 200 \mathrm{~T}$, and hence, one can exclude this kind of intrinsic pinning. Assuming instead $n$ equally spaced vortex rows across the film thickness leads to $B_{0}=2(n+1) \Phi_{0} /\left(\sqrt{3} \gamma t^{2}\right)$, where $t=22 \mathrm{~nm}$ is the film thickness as determined by TEM. The lowest possible matching field configuration is a single row in the center of the film due to the strong Bean-Livingston barriers ${ }^{60}$ on both interfaces (i.e. substrate/NdFeAs(O,F) and $\mathrm{NdOF} / \mathrm{NdFeAs}(\mathrm{O}, \mathrm{F}))$. In that case, and assuming the relevant anisotropy parameter is $\gamma_{J}(T)$ (Fig. 8), the temperature-dependent matching field position is shown in the inset of Fig. 8f and corresponds nicely to the peak positions in $N(H)$. This can be explained in terms of the most stable vortex configuration where excited structures such as kinks and double kinks as well as displacements are most effectively suppressed leading to reduced creep rates $S$ and consequently to a maximum in $N$. On the contrary, for around half filling of such a single row (at $B_{0} / 2$ ), a minimum in $N(H)$ can be observed since such excited structures and therefore creep are most probable around these fields. Higher orders of vortex row configurations may be guessed for $n=2$ or 3 but cannot be observed unambiguously due to increased noise levels at low $J_{\mathrm{c}}$ and $N$. For thicker films this matching effect has not been observed. For the $60 \mathrm{~nm}$ film in ref. 12, e.g., the peak is expected at $B_{0}=0.39 \ldots 0.44 \mathrm{~T}$ for temperatures of $4.2 \ldots 30 \mathrm{~K}$. In this field range, $N(B)$ is much steeper and the scatter in $N$ is larger due to the lower number of data points in $V(I)$ and $N(B)$. A possibly larger surface roughness of the $60 \mathrm{~nm}$ film as well as a larger freedom of the vortices to rearrange across the film thickness would further wash out such matching effects.

These matching effects, in combination with the strong pinning by the nanoscale inhomogeneities (Fe-rich, Fe-As, $\mathrm{NdFeO}$ precipitates) and potentially the magnetic contribution to pinning by the Fe particles lead to the observed superior pinning force densities and in-field critical current densities in this $22 \mathrm{~nm} \operatorname{NdFeAs}(\mathrm{O}, \mathrm{F})$ film, especially for $H \| a b$.

\section{Conclusions}

The structural and electrical properties of a superconducting epitaxial $22 \mathrm{~nm} \mathrm{NdFeAs}\left(\mathrm{O}_{1-x} \mathrm{~F}_{x}\right)$ thin film on a MgO single crystalline substrate were analyzed. Near-atomic-resolution measurements with TEM and APT reveal nanoscale inhomogeneities in the element distribution. The thin film contains small Fe nanoparticles and other Fe- and O-rich phases of different size, shape and orientation distribution. The electrical transport properties were measured in high magnetic fields. The interplay between small nanoparticles, $a b$-planar pinning centers, and intrinsic pinning as well as matching effects related to the film thickness leads to high $J_{\mathrm{c}}\left(J_{\mathrm{c}, \mathrm{sf}} \approx 7.6 \mathrm{MA} \mathrm{cm}^{-2}\right.$ at $\left.4.2 \mathrm{~K}\right)$ and $F_{\mathrm{p}} \sim 1$ $\mathrm{TN} \mathrm{m}^{-3}$ for $H \| a b(4.2 \mathrm{~K}, 35 \mathrm{~T})$ respectively. The angular dependence of $H_{\mathrm{c} 2}(\theta)$ is influenced by the multi-band superconductivity which also influences $J_{\mathrm{c}}(\theta)$ where $J_{\mathrm{c}}$ can be scaled in a wide angular range by the Anisotropic Ginzburg-Landau approach.

\section{Conflicts of interest}

There are no conflicts to declare.

\section{Author contributions}

SKW, KI and JH designed the study. SKW, TO, TM and TH prepared the films by molecular beam epitaxy (MBE) at Nagoya University. SM, CT, JJ and KI measured the high-field properties up to $35 \mathrm{~T}$ at NHMFL Tallahassee. CT, SKW, SM and JH measured and analysed further in-field transport data up to 14 (at KIT) and $16 \mathrm{~T}$ (at NHMFL). TB took care of the atom probe tomography (APT) investigations, RS and DG the transmission electron microscopy (TEM) studies. SKW and ML investigated the microstructure by X-ray diffraction (XRD). $\mathrm{HI}$ and $\mathrm{BH}$ supervised parts of the project. The manuscript was written by SKM together with KI, JH and CT, where all co-authors contributed to style and content at different stages of writing.

\section{Acknowledgements}

The authors thank Dominic Barthlott (KIT, ITEP) for experimental support and Delphine Chassaing (KIT, IAM-WK) for the FIB preparation of APT samples. This work was supported by the JSPS Grant-in-Aid for Scientific Research (B) Grant Number 16H04646 as well as JST CREST Grant Number JPMJCR18J4. A portion of this work was performed at the National High Magnetic Field Laboratory, which is supported by National Science Foundation Cooperative Agreement No. DMR-1644779, DMR-1157490 and the State of Florida.

\section{Notes and references}

1 N. R. Werthamer, E. Helfand and P. C. Hohenberg, Temperature and Purity Dependence of the Superconducting Critical Field, Hc2 III. Electron Spin and Spin-Orbit Effects, Phys. Rev., 1966, 147, 295-302, DOI: 10.1103/PhysRev.147.295.

2 J.-l. Zhang, L. Jiao, Y. Chen and H.-q. Yuan, Universal behavior of the upper critical field in iron-based superconductors, Front. Phys., 2011, 6, 463-473, DOI: 10.1007/s11467-011-0235-7.

3 J. Hänisch, K. Iida, R. Hühne and C. Tarantini, Fe-based superconducting thin films - preparation and tuning of superconducting properties, Supercond. Sci. Technol., DOI: 10.1088/1361-6668/ab1c00.

4 M. Fujioka, S. J. Denholme, T. Ozaki, H. Okazaki, K. Deguchi, S. Demura, H. Hara, T. Watanabe, H. Takeya, T. Yamaguchi, H. Kumakura and Y. Takano, Phase diagram and superconductivity at $58.1 \mathrm{~K}$ in $\alpha$-FeAs-free $\mathrm{SmFeAsO}_{1-\mathrm{x}} \mathrm{F}_{\mathrm{x}}$, Supercond. Sci. Technol., 2013, 26, 085023, DOI: 10.1088/ 0953-2048/26/8/085023.

5 S. Takeda, S. Ueda, S. Takano, A. Yamamoto and M. Naito, Growth of superconducting $\operatorname{SmFeAs}(\mathrm{O}, \mathrm{F})$ epitaxial films by F diffusion, Supercond. Sci. Technol., 2012, 25, 035007, DOI: 10.1088/0953-2048/25/3/035007.

6 A. L. Ivanovskii, New high-temperature superconductors based on rare-earth and transition metal oxyarsenides and related phases: synthesis, properties and simulations, Phys.-Usp., 2008, 51, 1229-1260, DOI: 10.1070/ PU2008v051n12ABEH006703. 
7 R. Kappenberger, S. Aswartham, F. Scaravaggi, C. G. F. Blum, M. I. Sturza, A. U. B. Wolter, S. Wurmehl and B. Büchner, Solid state single crystal growth of three-dimensional faceted LaFeAsO crystals, J. Cryst. Growth, 2018, 483, 9-15, DOI: 10.1016/j.jcrysgro.2017.11.006.

8 J. Karpinski, N. D. Zhigadlo, S. Katrych, Z. Bukowski, P. J. W. Moll, S. Weyeneth, H. Keller, R. Puzniak, M. Tortello, D. Daghero, R. Gonnelli, I. Maggio-Aprile, Y. Fasano, $\emptyset$. Fischer, K. Rogacki and B. Batlogg, Single crystals of $\mathrm{LnFeAsO}_{1-\mathrm{x}} \mathrm{F}_{\mathrm{x}}(\mathrm{Ln}=\mathrm{La}, \mathrm{Pr}, \mathrm{Nd}, \mathrm{Sm}, \mathrm{Gd})$ and $\mathrm{Ba}_{1-\mathrm{x}} \mathrm{Rb}_{\mathrm{x}} \mathrm{Fe}_{2} \mathrm{As}_{2}$ : Growth, structure and superconducting properties, Phys. C, 2009, 469, 370-380, DOI: 10.1016/j.physc.2009.03.048.

9 H. Hiramatsu, T. Katase, T. Kamiya and H. Hosono, Thin Film Growth and Device Fabrication of Iron-Based Superconductors, J. Phys. Soc. Jpn., 2012, 81, 011011, DOI: 10.1143/JPSJ.81.011011.

10 M. Chihara, N. Sumiya, K. Arai, A. Ichinose, I. Tsukada, T. Hatano, K. Iida and H. Ikuta, Direct growth of superconducting $\mathrm{NdFeAs}(\mathrm{O}, \mathrm{F})$ thin films by MBE, Phys. $C$, 2015, 518, 69-72, DOI: 10.1016/j.physc.2015.03.022.

11 K. Iida, J. Hänisch, C. Tarantini, F. Kurth, J. Jaroszynski, S. Ueda, M. Naito, A. Ichinose, I. Tsukada, E. Reich, V. Grinenko, L. Schultz and B. Holzapfel, Oxypnictide SmFeAs(O,F) superconductor: a candidate for high-field magnet applications, Sci. Rep., 2013, 3, 2139, DOI: 10.1038/ srep02139.

12 C. Tarantini, K. Iida, J. Hänisch, F. Kurth, J. Jaroszynski, N. Sumiya, M. Chihara, T. Hatano, H. Ikuta, S. Schmidt, P. Seidel, B. Holzapfel and D. C. Larbalestier, Intrinsic and extrinsic pinning in $\mathrm{NdFeAs}(\mathrm{O}, \mathrm{F})$ : vortex trapping and lockin by the layered structure, Sci. Rep., 2016, 6, 36047, DOI: 10.1038/srep36047.

13 M. Kidszun, S. Haindl, T. Thersleff, J. Hänisch, A. Kauffmann, K. Iida, J. Freudenberger, L. Schultz and B. Holzapfel, Critical Current Scaling and Anisotropy in Oxypnictide Superconductors, Phys. Rev. Lett., 2011, 106, 137001, DOI: 10.1103/PhysRevLett.106.137001.

14 P. J. W. Moll, R. Puzniak, F. F. Balakirev, K. Rogacki, J. Karpinski, N. D. Zhigadlo and B. Batlogg, High magneticfield scales and critical currents in $\operatorname{SmFeAs}(\mathrm{O}, \mathrm{F})$ crystals, Nat. Mater., 2010, 9, 628-633, DOI: 10.1038/nmat2795.

15 H. Uemura, T. Kawaguchi, T. Ohno, M. Tabuchi, T. Ujihara, Y. Takeda and H. Ikuta, Substrate dependence of the superconducting properties of $\operatorname{NdFeAs}(\mathrm{O}, \mathrm{F})$ thin films, Solid State Commun., 2012, 152, 735-739, DOI: 10.1016/ j.ssc.2012.01.037.

16 T. Kawaguchi, H. Uemura, T. Ohno, M. Tabuchi, T. Ujihara, Y. Takeda and H. Ikuta, Molecular Beam Epitaxy Growth of Superconducting NdFeAs(O,F) Thin Films Using a F-Getter and a Novel F-Doping Method, Appl. Phys. Express, 2011, 4, 083102, DOI: 10.1143/APEX.4.083102.

17 D. J. Larson, T. J. Prosa, R. M. Ulfig, B. P. Geiser and T. F. Kelly, Local Electrode Atom Probe Tomography. A User's Guide, Springer New York, New York, NY, s.l., 2013.

18 G. Rupp, E. Springer and S. Roth, Critical current of multifilamentary Nb3Sn conductors up to $18 \mathrm{t}$, Cryogenics, 1977, 17, 141-144, DOI: 10.1016/0011-2275(77)90273-9.
19 M. Suenaga and D. O. Welch, in Filamentary A15 Superconductors, ed. M. Suenaga and A. F. Clark, Springer, Boston, MA, 1980, pp. 131-142.

20 J. B. Nelson and D. P. Riley, An experimental investigation of extrapolation methods in the derivation of accurate unit-cell dimensions of crystals, Proc. Phys. Soc., 1945, 57, 160-177, DOI: $10.1088 / 0959-5309 / 57 / 3 / 302$.

21 P. A. Stadelmann, EMS - a software package for electron diffraction analysis and HREM image simulation in materials science, Ultramicroscopy, 1987, 21, 131-145, DOI: 10.1016/0304-3991(87)90080-5.

22 Y. Zhang, C. T. Nelson, S. Lee, J. Jiang, C. W. Bark, J. D. Weiss, C. Tarantini, C. M. Folkman, S.-H. Baek, E. E. Hellstrom, D. C. Larbalestier, C.-B. Eom and X. Pan, Self-assembled oxide nanopillars in epitaxial $\mathrm{BaFe}_{2} \mathrm{As}_{2}$ thin films for vortex pinning, Appl. Phys. Lett., 2011, 98, 42509, DOI: $10.1063 / 1.3532107$.

23 F. Kurth, C. Tarantini, V. Grinenko, J. Hänisch, J. Jaroszynski, E. Reich, Y. Mori, A. Sakagami, T. Kawaguchi, J. Engelmann, L. Schultz, B. Holzapfel, H. Ikuta, R. Hühne and K. Iida, Unusually high critical current of clean P-doped $\mathrm{BaFe}_{2} \mathrm{As}_{2}$ single crystalline thin film, Appl. Phys. Lett., 2015, 106, 072602, DOI: 10.1063/ 1.4908257.

24 S. Haindl, K. Hanzawa, H. Sato, H. Hiramatsu and H. Hosono, In-situ growth of superconducting $\mathrm{SmO}_{1-\mathrm{x}} \mathrm{F}_{\mathrm{x}}$ FeAs thin films by pulsed laser deposition, Sci. Rep., 2016, 6, 3296, DOI: 10.1038/srep35797.

25 Z. Pribulova, T. Klein, J. Kacmarcik, C. Marcenat, M. Konczykowski, S. L. Bud'ko, M. E. Tillman and P. C. Canfield, Upper and lower critical magnetic fields of superconducting $\mathrm{NdFeAsO}_{1-\mathrm{x}} \mathrm{F}_{\mathrm{x}}$ single crystals studied by Hall-probe magnetization and specific heat, Phys. Rev. B, 2009, 79, 020508(R), DOI: 10.1103/PhysRevB.79.020508.

26 W. E. Lawrence and S. Doniach, Theory of Layer Structure Superconductors, Proceedings of the Twelfth International Conference on Low Temperature Physics, 1971, pp. 361-362.

27 G. Blatter, M. V. Feigel'man, V. B. Geshkenbein, D. J. Larkin and V. M. Vinokur, Vortices in high-temperature superconductors, Rev. Mod. Phys., 1994, 66, 1126-1388.

28 L. Fang, Y. Jia, V. Mishra, C. Chaparro, V. K. Vlasko-Vlasov, A. E. Koshelev, U. Welp, G. W. Crabtree, S. Zhu, N. D. Zhigadlo, S. Katrych, J. Karpinski and W. K. Kwok, Huge critical current density and tailored superconducting anisotropy in $\mathrm{SmFeAsO}_{0.8} \mathrm{~F}_{0.15}$ by low-density columnardefect incorporation, Nat. Commun., 2013, 4, 3296, DOI: 10.1038/ncomms3655.

29 A. K. Ghosh, M. Tokunaga and T. Tamegai, Angular dependence of the upper critical field in CaAlSi single crystal: Deviation from the Ginzburg-Landau anisotropic mass model, Phys. Rev. B: Condens. Matter Mater. Phys, 2003, 68, 63, DOI: 10.1103/PhysRevB.68.054507.

30 J. Hänisch, K. Iida, F. Kurth, E. Reich, C. Tarantini, J. Jaroszynski, T. Förster, G. Fuchs, R. Hühne, V. Grinenko, L. Schultz and B. Holzapfel, High field superconducting properties of $\mathrm{Ba}\left(\mathrm{Fe}_{1-\mathrm{x}} \mathrm{Co}_{\mathrm{x}}\right)_{2} \mathrm{As}_{2}$ thin films, Sci. Rep., 2015, 5, 17363, DOI: 10.1038/srep17363. 
31 K. Iida, H. Sato, C. Tarantini, J. Hänisch, J. Jaroszynski, H. Hiramatsu, B. Holzapfel and H. Hosono, High-field transport properties of a P-doped $\mathrm{BaFe}_{2} \mathrm{As}_{2}$ film on technical substrate, Sci. Rep., 2017, 7, 39951, DOI: 10.1038/ srep39951.

32 E. F. Talantsev and R. C. Mataira, Polar projections for big data analysis in applied superconductivity, AIP Adv., 2018, 8, 75213, DOI: 10.1063/1.5038040.

33 S. Galeski, P. J. W. Moll, N. D. Zhigadlo, K. Mattenberger and B. Batlogg, Critical fields and fluctuations determined from specific heat and magnetoresistance in the same nanogram SmFeAs(O,F) single crystal, Phys. Rev. B, 2017, 96, 104511, DOI: $10.1103 /$ PhysRevB.96.104511.

34 M. Tinkham, Effect of Fluxoid Quantization on Transitions of Superconducting Films, Phys. Rev., 1963, 129, 24132422, DOI: 10.1103/PhysRev.129.2413.

35 R. Fastampa, M. Giura, R. Marcon and E. Silva, 2D to 3D crossover in Bi-Sr-Ca-Cu-O: comparison with synthetic multilayered superconductors, Phys. Rev. Lett., 1991, 67, 1795-1798, DOI: 10.1103/PhysRevLett.67.1795.

36 R. Marcon, R. Fastampa, M. Giura and E. Silva, Anisotropy and Dimensionality in the Resistive Measurements in Epitaxial Films of BiSrCaCuO, Europhys. Lett., 1991, 16, 757-762, DOI: 10.1209/0295-5075/16/8/009.

37 J. R. Thompson, K. D. Sorge, C. Cantoni, H. R. Kerchner, D. K. Christen and M. Paranthaman, Vortex pinning and slow creep in high- $\mathrm{J}_{\mathrm{c}} \mathrm{MgB}_{2}$ thin films: a magnetic and transport study, Supercond. Sci. Technol., 2005, 18, 970-976, DOI: 10.1088/0953-2048/18/7/008.

38 J. Hänisch, K. Iida, T. Ohmura, T. Matsumoto, T. Hatano, M. Langer, S. Kauffmann-Weiss, H. Ikuta and B. Holzapfel, Vortex glass-liquid transition and activated flux motion in an epitaxial, superconducting $\operatorname{NdFeAs}(\mathrm{O}, \mathrm{F})$ thin film, Magn. Reson. Chem., 2018, 47, 1-6, DOI: 10.1557/ mrc.2018.207.

39 C. Tarantini, F. Kametani, S. Lee, J. Jiang, J. D. Weiss, J. Jaroszynski, E. E. Hellstrom, C.-B. Eom and D. C. Larbalestier, Development of very high $\mathrm{J}_{\mathrm{c}}$ in $\mathrm{Ba}\left(\mathrm{Fe}_{1-}\right.$ $\left.{ }_{\mathrm{x}} \mathrm{Co}_{\mathrm{x}}\right)_{2} \mathrm{As}_{2}$ thin films grown on $\mathrm{CaF}_{2}, \mathrm{Sci}$. Rep., 2014, 4, 7305, DOI: 10.1038/srep07305.

40 A. Sakagami, T. Kawaguchi, M. Tabuchi, T. Ujihara, Y. Takeda and H. Ikuta, Critical current density and grain boundary property of $\mathrm{BaFe}_{2}(\mathrm{As}, \mathrm{P})_{2}$ thin films, Phys. $C$, 2013, 494, 181-184, DOI: 10.1016/j.physc.2013.04.047.

41 A. Gurevich, Pinning size effects in critical currents of superconducting films, Supercond. Sci. Technol., 2007, 20, S128-S135, DOI: 10.1088/0953-2048/20/9/S03.

42 G. Blatter, V. B. Geshkenbein and D. J. Larkin, From isotropic to anisotropic superconductors: a scaling approach, Phys. Rev. Lett., 1992, 68, 875-878, DOI: 10.1103/ PhysRevLett.68.875.

43 L. Civale, B. Maiorov, A. Serquis, J. O. Willis, J. Y. Coulter, H. Wang, Q. X. Jia, P. N. Arendt, M. Jaime, J. L. MacManus-Driscoll, M. P. Maley and S. R. Foltyn, Understanding high critical currents in $\mathrm{YBa}_{2} \mathrm{Cu}_{3} \mathrm{O}_{7}$ thin films and coated conductors, J. Low Temp. Phys., 2004, 135, $87-98$.
44 K. Iida, J. Hänisch, T. Thersleff, F. Kurth, M. Kidszun, S. Haindl, R. Hühne, L. Schultz and B. Holzapfel, Scaling behavior of the critical current in clean epitaxial $\mathrm{Ba}\left(\mathrm{Fe}_{1-\mathrm{x}} \mathrm{Co}_{\mathrm{x}}\right)_{2} \mathrm{As}_{2}$ thin films, Phys. Rev. B: Condens. Matter Mater. Phys., 2010, 81, 100507R, DOI: 10.1103/ PhysRevB.81.100507.

45 J. D. Fletcher, A. Carrington, O. J. Taylor, S. M. Kazakov and J. Karpinski, Temperature-dependent anisotropy of the penetration depth and coherence length of $\mathrm{MgB}_{2}$, Phys. Rev. Lett., 2005, 95, 097005, DOI: 10.1103/ PhysRevLett.95.097005.

46 S. Weyeneth, R. Puzniak, N. D. Zhigadlo, S. Katrych, Z. Bukowski, J. Karpinski and H. Keller, Evidence for Two Distinct Anisotropies in the Oxypnictide Superconductors $\mathrm{SmFeAsO}_{0.8} \mathrm{~F}_{0.2}$ and $\mathrm{NdFeAsO}_{0.8} \mathrm{~F}_{0.2}$, J. Supercond. Novel Magn., 2009, 22, 347-351, DOI: 10.1007/s10948-009-0445-1.

47 K. Iida, J. Hänisch, E. Reich, F. Kurth, R. Hühne, L. Schultz, B. Holzapfel, A. Ichinose, M. Hanawa, I. Tsukada, M. Schulze, S. Aswartham, S. Wurmehl and B. Büchner, Intrinsic pinning and the critical current scaling of clean epitaxial Fe(Se,Te) thin films, Phys. Rev. B: Condens. Matter Mater. Phys., 2013, 87, 104510, DOI: 10.1103/ PhysRevB.87.104510.

48 W. H. Warnes and D. C. Larbalestier, Analytical technique for deriving the distribution of critical currents in a superconducting wire, Appl. Phys. Lett., 1986, 48, 14031405, DOI: 10.1063/1.96923.

49 R. Griessen, Relaxation effects, I-V curves and irreversibility lines in high-Tc superconductors, Phys. C, 1991, 175, 315323, DOI: 10.1016/0921-4534(91)90603-V.

$50 \mathrm{H}$. Yamasaki and Y. Mawatari, Current-voltage characteristics of melt-textured YBCO obtained from the field-sweep rate dependence of magnetization, IEEE Trans. Appl. Supercond., 1999, 9, 2651-2654, DOI: 10.1109/ 77.785031.

51 J. R. Thompson, Ö. Polat, D. K. Christen, D. Kumar, P. M. Martin and J. W. Sinclair, Wide-range characterization of current conduction in high-Tc coated conductors, Appl. Phys. Lett., 2008, 93, 042506, DOI: 10.1063/1.2964195.

52 L. Opherden, M. Sieger, P. Pahlke, R. Hühne, L. Schultz, A. Meledin, G. van Tendeloo, R. Nast, B. Holzapfel, M. L. Bianchetti, J. L. MacManus-Driscoll and J. Hänisch, Large pinning forces and matching effects in $\mathrm{YBa}_{2} \mathrm{Cu}_{3} \mathrm{O}_{7^{-}}$ d thin films with $\mathrm{Ba}_{2} \mathrm{Y}(\mathrm{Nb} / \mathrm{Ta}) \mathrm{O}_{6}$ nano-precipitates, Sci. Rep., 2016, 6, 21188.

53 J. H. Kim, S. X. Dou, A. Matsumoto, S. Choi, T. Kiyoshi and H. Kumakura, Correlation between critical current density and $\mathrm{n}$-value in $\mathrm{MgB}_{2} / \mathrm{Nb} /$ Monel superconductor wires, Phys. C, 2010, 470, 1207-1210, DOI: 10.1016/ j.physc.2010.05.075.

54 D. M. J. Taylor and D. P. Hampshire, Relationship between the $\mathrm{n}$-value and critical current in $\mathrm{Nb}_{3} \mathrm{Sn}$ superconducting wires exhibiting intrinsic and extrinsic behavior, Supercond. Sci. Technol., 2005, 18, S297-S302.

55 G. Manfreda, F. Bellina and G. Volpini, Critical current statistical distribution and voltage-current characteristics 
in superconducting wires, Supercond. Sci. Technol., 2014, 27, 125005.

56 P. Paturi, M. Malmivirta, H. Palonen and H. Huhtinen, Dopant diameter dependence of $\mathrm{Jc}(\mathrm{B})$ in doped $\mathrm{YBCO}$ films, IEEE Trans. Appl. Supercond., 2016, 26, 8000705, DOI: 10.1109/TASC.2016.2533567.

57 D. Dew-Hughes, Flux pinning mechanisms in type II superconductors, Philos. Mag., 1974, 30, 293-305, DOI: 10.1080/14786439808206556.
58 E. J. Kramer, Scaling laws for flux pinning in hard superconductors, J. Appl. Phys., 1973, 44, 1360-1370, DOI: 10.1063/1.1662353.

59 M. G. Blamire, R. B. Dinner, S. C. Wimbush and J. L. MacManus-Driscoll, Critical current enhancement by Lorentz force reduction in superconductor-ferromagnet nanocomposites, Supercond. Sci. Technol., 2009, 22, 025017, DOI: 10.1088/0953-2048/22/2/025017.

60 C. P. Bean and J. D. Livingston, Surface Barrier in Type-II Superconductors, Phys. Rev. Lett., 1964, 12, 14-16, DOI: 10.1103/PhysRevLett.12.14. 